\title{
Performance Degradation of Ferrofluidic Feedthroughs in a Mixed Irradiation Field
}

\author{
Nikolaos Simos $^{1 *}$, S. Fernandes ${ }^{2}$, Wolfgang Mittig ${ }^{2,3}$, Frederique Pellemoine ${ }^{2}$ \\ M. Avilov ${ }^{2}$, M. Kostin ${ }^{2}$, L. Mausner ${ }^{1}$, R. Ronningen ${ }^{2}$, M. Schein ${ }^{2}$, G. Bollen ${ }^{2}$ \\ (1) Brookhaven National Laboratory BNL, Upton NY 11973, USA \\ ${ }^{(2)}$ Facility for Rare Isotope Beams (FRIB), Michigan State University, \\ East Lansing MI 48824, USA \\ (3) National Superconducting Cyclotron Laboratory NSCL, Michigan State University, \\ 640 South Shaw Lane, East Lansing MI 48824-1321, USA
}

\begin{abstract}
Ferrofluidic feedthrough (FF) rotary seals containing either NdFeB or SmCo-type permanent magnets have been considered for use in the target and beam dump systems of the Facility for Rare Isotope Beams (FRIB). To evaluate their performance under irradiation three FF seals were irradiated in a mixed field consisting of fast neutrons, protons and $\gamma$-rays to an average absorbed dose of 0.2, 2.0, and 20.0 MGy at the Brookhaven Linac Isotope Producer facility (BLIP). The radiation types and energy profiles mimic those expected at the FRIB facility. Degradation of the operational performance of these devices due to irradiation is expected to be the result of the de-magnetization of the permanent magnets contained within the seal and the changes in the ferrofluid properties. Post-irradiation performance was evaluated by determining the ferrofluidic seal vacuum tightness and torque under static and dynamic conditions. The study revealed that the ferrofluidic feedthrough seal irradiated to a dose of 0.2 MGy maintained its vacuum tightness under both static and rotational condition while the one irradiated to a dose of $2.0 \mathrm{MGy}$ exhibited signs of ferrofluid damage but no overall performance loss. At 20 MGy dose the effects of irradiation on the ferrofluid properties (viscosity and particle agglomeration) were shown to be severe. Furthermore, limited de-magnetization of the annular shaped $\mathrm{Nd}_{2} \mathrm{Fe}_{14} \mathrm{~B}$ and $\mathrm{Sm}_{2} \mathrm{Co}_{27}$ magnets located within the irradiated FFs was observed for doses of 0.2 MGy and 20 MGy respectively.
\end{abstract}

Keywords: Neutron irradiation, de-magnetization, radiation damage, ferrofluidic feedthrough, performance, static torque, rotational torque, leak rate.

Corresponding author: N. Simos, BNL; simos@bnl.gov; Tel: +1 6313447229 
Simos et al: Manuscript for Nuclear Instruments and Methods

\section{Introduction}

Ferrofluidic feedthrough (FF) seals capable of providing transmission of rotary motion through a vacuum boundary with essentially zero leakage at high speed and with low friction torque have been under consideration for use in the future projectile fragment separator of the Facility for Rare Isotope Beams (FRIB) [1-2]. The potential use of such feedthroughs was explored during the early stages of the project where they were to be integrated with the rare isotope production target [2] and the beam dump systems. The FRIB isotope target and dump systems are designed to support efficient operation of the facility under extreme conditions. Both systems must have a stable performance over their expected lifetime of two weeks and one year respectively under irradiation by intense heavy ion primary beams from oxygen to uranium having maximum beam power of $400 \mathrm{~kW}$. Therefore, the radiation "hardness" of the FF components is a critical parameter for the design of these systems due to the fact that they will be exposed to an intense mixed secondary radiation field consisting of neutrons, $\gamma$-rays, electrons and fragments.

The primary operational concern is the radiation-induced degradation of the FF's vulnerable components manifested in the form of breakdown of the magnetic ferrofluid and bearing lubricant, de-magnetization of the integrated permanent magnet, and degradation of the O-rings. Such effects will inevitably lead to static and rotational torque increase and eventually to seal leakage. The ferrofluid within the FF seal that enables the rotary motion across a vacuum boundary consists of colloidal suspensions of permanently magnetic particles [3-6] having magnetic moments of $\sim 10^{4} \mu \mathrm{B}$ in a carrier fluid such as oil or water. Irradiation-induced breakdown of the carrier fluid and/or particle agglomeration are areas of concern.

A campaign to assess the irradiation-induced degradation of three FF seals containing two types of permanent magnets, namely $\mathrm{Nd}_{2} \mathrm{Fe}_{14} \mathrm{~B}$ and $\mathrm{Sm}_{2} \mathrm{Co}_{14}$, was launched at BLIP by utilizing the mixed irradiation field, which consists of fast neutrons, protons and $\gamma$-rays. The goal was to achieve average absorbed doses of 0.2, 2.0, and 20.0 MGy. The irradiating species and their energy distributions at the selected experimental configuration at BLIP approximately mimic those expected at the actual set-up at the FRIB facility. The 20.0 MGy dose was correspond to the estimated maximum exposure of a feedthrough installed in the isotope target system during FRIB operation with a $266 \mathrm{MeV}{ }^{18} \mathrm{O}$ beam having $400 \mathrm{~kW}$ power for a month-long run without radiation shield. The effect of irradiation on the operational performance of these devices influenced by resistance to de-magnetization and to ferrofluid property changes were evaluated by determining the ferrofluidic seal vacuum tightness and torque under static and dynamic conditions.

Several radiation damage studies with thermal neutrons and $\gamma$-ray exposure have been performed with FF seals and respective material components to determine their threshold radiation dose limit [4, 6-12]. In spite of these studies the effects of radiation on the chemical and physical properties of ferrofluids are still not yet well understood. Changes in the viscosity, surface tension and colloid stability of ester-based ferrofluid (manufactured by Rigaku Co.) were studied [7] following irradiation using a $\gamma$-ray source for up to $1.8 \mathrm{MGy}$ dose and these were found to be negligible. Kopčanský and co-workers [12] also studied the effect of $\gamma$-radiation on the magnetization of kerosene-based ferrofluid and observed that (a) the saturation magnetization and number of magnetic particles both decrease with increased $\gamma$-irradiation dose and (b) $\gamma$ radiation induces agglomeration and sedimentation with increased dose. It was assessed [12] that radiation induces breakdown of long molecular chains in the carrier fluid resulting in the acceleration of agglomeration and subsequently sedimentation. Nanoscale gadolinium oxide $\left(\mathrm{Gd}_{2} \mathrm{O}_{3}\right)$ based ferrofluids coated with synthesized surfactant were $\gamma$-ray irradiated to doses in the range of $32 \mathrm{~Gy}-2.635 \mathrm{kGy}$ [13], and with the help of high-resolution transmission electron microscope (HRTEM) it was determined that the particles developed intergranular defects. Embs and co-workers [14] studied the spatiotemporal behavior of equilibrium and non-equilibrium properties of ferrofluids in various magnetic fields. Specifically, [14] assessed the instability behavior of ferrofluids by comparing experimental results to analytical calculations and numerical approaches. Of direct relevance to the present study, they experimentally quantified the influence on the rotational dynamics of the nano-scaled magnetic particles 
in the ferrofluid by a macroscopic flow (such as rigid rotation) and/or by an external magnetic field. An overview of studies on the magneto-rheology of ferrofluids with emphasis in the understanding of the nature of field-induced microstructure in ferrofluids and the various factors affecting their magnetorheological behavior is presented in [15].

De-magnetization effects by various types of radiation fields, including neutrons, protons, ions, electrons, and $\gamma$-rays, on $\mathrm{Nd}_{2} \mathrm{Fe}_{14} \mathrm{~B}$ and $\mathrm{Sm}_{2} \mathrm{Co}_{27}$ magnets configured to have various orientations to the radiation field were studied in [16-21] revealing the hierarchical damage potential of the different irradiating species, the contributing role of irradiation temperature and of the orientation of the magnetic field relative to the direction of the radiation field. The resistance to de-magnetization stemming from the rare earth magnet microstructure, i.e. coarse vs. fine-grained, is also discussed and assessed [19].

Presented in the subsequent sections of this paper are the details of the irradiation experiments at BLIP and the results of the post-irradiation evaluation of the performance of the three irradiated FFs.

\section{Experimental}

\subsection{Ferrofluidic feedthrough Characterization}

A ferrofluid vacuum seal rotary feedthrough is an assembly of different components shown in Figure 1 consisting of a stainless steel housing, shaft, two pole pieces, a permanent magnet, two ball bearing sleeves, several ferrofluid "O-rings" and three static O-rings.
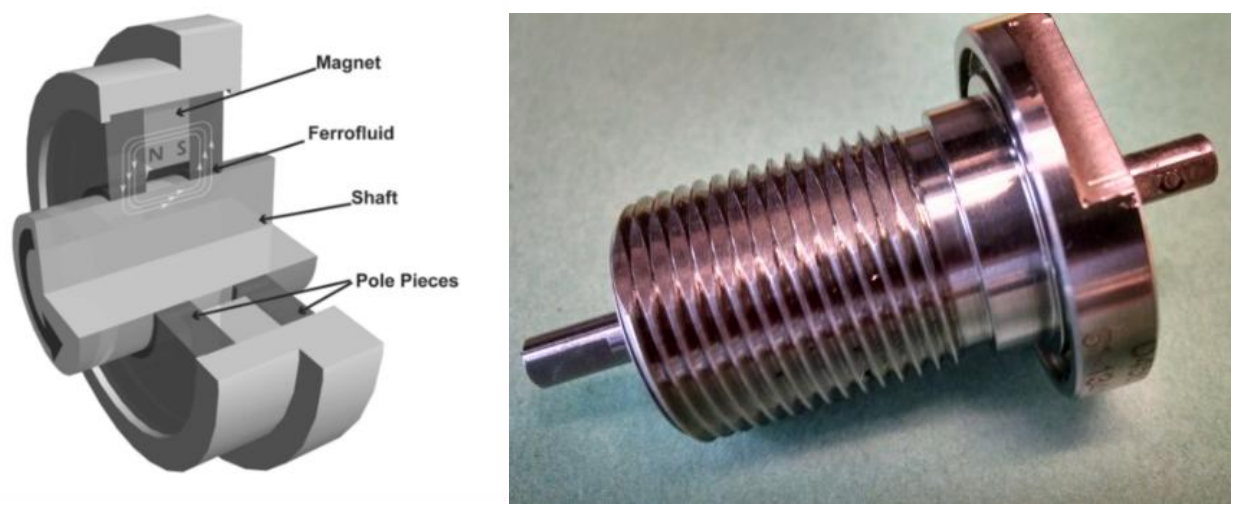

Figure 1: Graphics of a ferrofluidic feedthrough seal reproduced and adapted from [3].

A stationary ring-shaped permanent magnet encircles a rotating shaft that is sealed by multiple annular ferrofluid filled "O" ring segments running from air side-to-vacuum side of the device. The FF function relies on multiple ferrofluidic seals to support the total required differential pressure. The ferrofluid resides in the small gaps between the shaft and pole magnet, and the magnetic field increases its apparent viscosity to the point of becoming a viscoelastic liquid. The saturation magnetization determines the pressure capacity of the seal and the viscosity determines the power consumed by the seal in operation [4]. The ferrofluid is a magnetically stable colloidal suspension, typically made of iron oxide nanoparticles coated with a surfactant, such as oleic acid, and suspended in an organic liquid carrier, a hydrocarbon, ester, fluorocarbon, silicone or perfluoropolyether [5] based oil. The composition of a typical ferrofluid is $\sim 5 \%$ magnetic solids, $\sim 10 \%$ surfactant and $85 \%$ liquid carrier, by volume. Surfactant acts as a stabilizing agent by preventing the attractive forces, i.e. van der Walls and magnetic forces, of materials even when a high intensity external magnetic field is applied. Upon application of such a magnetic field the magnetic moments of the particles align with the magnetic field lines. Ferrofluids typically consist of particles that may have different sizes and thus magnetic moments. The size of the particles in the range of $\sim 10 \mathrm{~nm}$ provides opposition against segregation due to gravity and common magnetic field gradients. To prevent aggregation due to van-der-Waals forces the magnetic nanoparticles are coated suitable surfactants. Macroscopically, ferrofluids appear as paramagnetic liquids 
and for this reason they are called super-paramagnetic. The large magnetic moments easily allow exerting significant magnetic forces and magnetic torques on the particles.

The FF seals used in this study (model SNL-006-NN) were supplied by Ferrotec Inc. with Ferrotec Ferrofluidic ${ }^{\circledR}$ Seals [6] and Viton ${ }^{\circledR}$ static O-rings. The housing, shaft and bearings are made of 300, 400 and 440 series stainless steel respectively. The internal reference associated to the ferrofluid composition is VSG-903, which corresponds to a ferrofluid with a density of $1.12 \mathrm{~g} \cdot \mathrm{cm}^{-3}$ made of mineral oil and

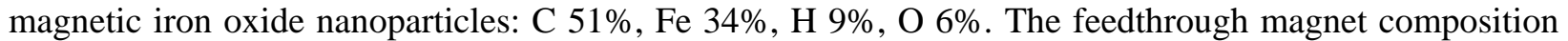
was selected according to the operational radiation dose environment requirements, either $\mathrm{Nd}_{2} \mathrm{Fe}_{14} \mathrm{~B}$ or $\mathrm{Sm}_{2} \mathrm{Co}_{17}$. $\mathrm{Sm}$-Co based magnets have superior thermal stability and radiation resistance as compared to $\mathrm{Nd}-\mathrm{Fe}-\mathrm{B}$ based magnets due to their higher magnetic coercivity and Curie temperature while the latter exhibit higher room-temperature magnetic properties [21]. Consequently improved performance at high radiation levels is expected by using $\mathrm{Sm}_{2} \mathrm{Co}_{17}$ magnets. For a dose of $0.2 \mathrm{MGy}$ the magnetic flux loss was estimated to be of the order of $2 \%$ [20], hence, the different magnet composition should have a negligible influence in the feedthrough performance at that dose. Therefore, the feedthrough irradiated to a dose of $0.2 \mathrm{MGy}$ was selected to have a $\mathrm{Nd}_{2} \mathrm{Fe}_{14} \mathrm{~B}$ magnet and the feedthroughs absorbing higher doses $(2$ and 20 MGy) contained $\mathrm{Sm}_{2} \mathrm{Co}_{17}$ magnets.

\subsection{Calculation of radiation dose level in the ferrofluidic seal for FRIB}

Detailed radiation transport calculations were performed to assess the dose levels in the FF seals considered in both the production target and the beam dump systems. For both the FRIB baseline and its energy upgrade option, the worst case scenario is represented by an oxygen beam of $400 \mathrm{~kW}$ power and energy of $266 \mathrm{MeV} / \mathrm{u}$ and $637 \mathrm{MeV} / \mathrm{u}$ respectively impinging on the production target and the beam dump [2]. The generated secondary beams will irradiate the components around the target and dump systems including the FF seals. Given that the total volume of ferrofluid for both FF seals considered is of the order of $1-2 \mathrm{~cm}^{3}$ the particle fluxes are expected to be uniform within the seal. The FFseal of the production target system was considered to be located at a minimal distance of about $50 \mathrm{~cm}$ opposite to the beam direction. The FF seal of the beam dump system was taken to be at $90^{\circ}$ with respect to the beam direction and at a distance of $120 \mathrm{~cm}$ from the beam interaction region in the dump.

Table 1 lists the estimated total radiation dose absorbed by the FF seals used in the target and beam dump systems when irradiated by secondary radiation produced by the ${ }^{18} \mathrm{O}$ beam, with or without radiation shielding. Calculations were done for production target at the FRIB baseline energy of $266 \mathrm{MeV} / \mathrm{u}$ and for beam dump at $637 \mathrm{MeV} / \mathrm{u}$ which conservatively represents the FRIB upgrade beam energy assumption.

Predictive calculations of dose for the planned irradiation of the three rotary FF seals at the BLIP target station that is dedicated to medical isotope production and material irradiation [16] were also performed. At the BLIP station a $118 \mathrm{MeV}$ proton beam with flux $\sim 6.0 \times 10^{14} \mathrm{p} / \mathrm{s}$ is stopped upstream of the FF seal by an array of isotope production targets made of rubidium chloride and gallium. A mixed secondary field produced by spallation and consisting of fast neutrons, protons, electrons and $\gamma$-rays irradiates the FF seal downstream (Figure 2). The predictive analyses revealed that at the irradiation location of the FF seal fast neutrons and high energy $\gamma$-rays dominate the mixed field and the estimated average absorbed dose rate during irradiation is $\sim 2.24 \mathrm{MGy} \cdot \mathrm{day}^{-1}$. In addition to the energy spectra of the irradiating species and dose rate, accumulated doses and energy depositions were also estimated using the MARS-15 [22] and the FLUKA [23, 24] transport codes.

Table 1 - Total radiation dose absorbed by the FF seal when target and beam dump are impacted by ${ }^{18} \mathrm{O}$ beams of energy $266 \mathrm{MeV} / \mathrm{u}$ and $637 \mathrm{MeV} / \mathrm{u}$ respectively 


\begin{tabular}{ccccc}
\hline System & $\begin{array}{c}{ }^{18} \mathrm{O} \text { beam energy } \\
(\mathrm{MeV} / \mathrm{u})\end{array}$ & $\begin{array}{c}\text { Total dose } \\
(\mathrm{MGy})\end{array}$ & Shielding & $\begin{array}{c}\text { Operation } \\
\text { lifetime (h) }\end{array}$ \\
\hline $\begin{array}{c}\text { Production } \\
\text { target }\end{array}$ & 266 & 7.5 & $\begin{array}{c}\text { None } \\
4 \mathrm{~cm} \text { of steel plus } \\
36 \mathrm{~cm} \text { of cast iron }\end{array}$ & 340 \\
\hline Beam dump & 637 & 3.5 & $13 \mathrm{~cm}$ of steel & 5500 \\
\hline
\end{tabular}

\subsection{Irradiation Experiment}

FF seal irradiation was performed within the spallation reaction-produced mixed field of fast neutrons, protons, electrons and $\gamma$-rays generated downstream of the isotope producing targets at BLIP (Figure 2). Specifically, the isotope target array stops the $118 \mathrm{MeV}$ primary proton beam from the Linac generating the mixed field profiles shown in Figure 3 (a-d). The irradiation was performed in a $9.3 \mathrm{~m}$ high column of cooling water flowing past the isotope target capsules and the FF seal. Irradiations were conducted under static conditions. The temperature rise due to the power deposition by the mixed field was estimated using the energy deposition predictions from the transport code analysis coupled with finite element-based thermal analysis procedures using the LS-DYNA code [25]. The thermal analyses studies showed $<10^{\circ} \mathrm{C}$ temperature rise in the FF seal. This implies that the FF seal irradiation temperature is well below the Curie temperatures for both the ferrofluid $\mathrm{Fe}_{3} \mathrm{O}_{4}\left(585^{\circ} \mathrm{C}\right)$ and the contained permanent magnets $\left(\mathrm{Sm}_{2} \mathrm{Co}_{17}\right.$ and $\mathrm{Nd}_{2} \mathrm{Fe}_{14} \mathrm{~B}$ ).

Photon spectra from all three irradiated FF seals were measured using a high sensitivity Ge detector at the End-of-Bombardment (EOB). Demagnetization of the permanent magnets within the FF seal was also measured promptly after EOB. A 6-month decay period necessitated by the high level of induced radioactivity was allowed between the end of irradiation and the post-irradiation evaluation of static and rotational torque as well as the leak rate measurements.

\section{Irradiation Conditions at the Ferrofluidic Feedthrough}

In an effort to deduce valid post-irradiation performance of the FF seals as a function of the irradiation levels while acknowledging the fact that performance degradation will result from two primary sources, i.e. demagnetization of the permanent magnet within the seal and the irradiation-induced changes in the ferrofluid, a precise representation of both the geometry of the FF seal and its orientation in the irradiating field and of the composition of the materials making up both the magnet and the ferrofluid was generated and utilized in the subsequent studies including those using radiation transport code systems such as FLUKA [23, 24]. This refined model was used to correlate results and other published data of radiationinduced demagnetization of permanent magnets and radiation-induced sedimentation of ferrofluids as a result of irradiation by different irradiating species, and finally correlate measured spectra of the FF seal as a whole or from outgassing during post-irradiation testing as an attempt to pinpoint the element mostly contributing to the performance degradation of the FF seal.

210

211

212

213

214

215

216

217
The global model that was developed to help evaluate the flux, fluence, energy spectra and dose rates for the different components in the FF seal is depicted in Figure 2. In the model the exact representation of the isotope target array (within Position 1as shown in Figure 2) upstream of the FF seal location that is responsible for generating the utilized mixed field, was precisely modeled to ensure that the field downstream is most representative. The FF seal located within Position 2 of the irradiation station at BLIP was modeled in detail to capture not only the components within the unit with different composition but with the components in the exact position within the irradiating mixed field. Specifically, as shown in 
Figure 2, the FF seals were placed in the vertical position and occupied the entirety of Position 2 in the

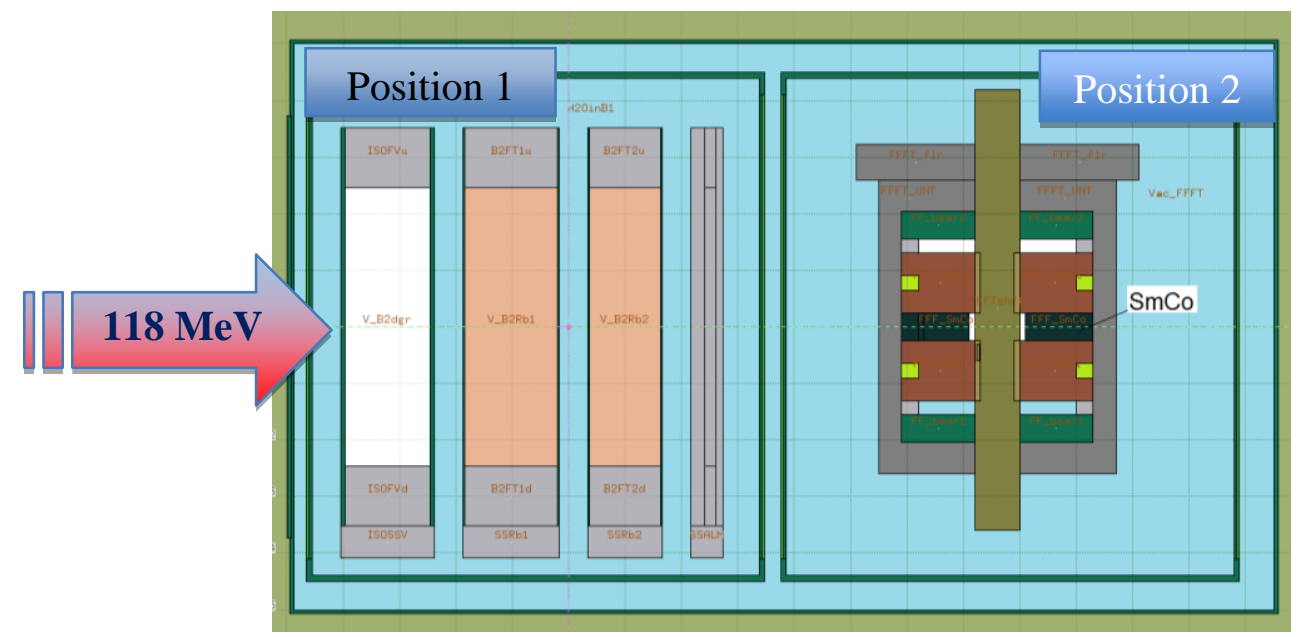

Figure 2: Detailed FLUKA model of irradiation station layout at BLIP including upstream spallation target configuration and FF orientation.

Therefore, the FF model developed and utilized in the FLUKA-based analysis included the stainless shell and shaft exact composition, the Viton O-rings, the aluminum spacer, the cores, the Sm-Co magnet, and the $2 \mathrm{~cm}^{3}$ ferrofluid volume. Given that one of the key outputs sought in the analysis is the isotopic signatures of the outgas products captured during the post-irradiation tests, special care was given to the compositional modeling of the ferrofluid. For these tests the Ferrotec Inc. VSG-903 model was used, consisting of $5 \%$ magnetic iron oxide nanoparticles (hematite $\mathrm{Fe}_{2} \mathrm{O}_{3}$ ), $10 \%$ surfactant $\left(\mathrm{CH}_{3}\left(\mathrm{CH}_{2}\right)_{11}\left(\mathrm{OCH}_{2} \mathrm{CH}_{2}\right)_{3} \mathrm{OSO}_{3} \mathrm{Mg}\right)$ and $85 \%$ mineral oil with an overall density of $1.12 \mathrm{~g} \cdot \mathrm{cm}^{-}{ }^{3}$.

Two (2) analyses were conducted. In the first analysis energy deposition, spallation product profiles over the FF seal, energy spectra at selected locations and dose profiles were estimated. In the second analysis the isotopic photon signatures of the induced radioactivity in different components (with focus on the SmCo magnet and the ferrofluid) for different decay times after irradiation were deduced. The predicted isotopic signatures were used to compare with the actual photon spectra measured. Figures 3 depict the neutron, $\gamma$ and electron spectra at the upstream face of the Sm-Co magnet. Figures 3(a,b,c,d) represent:

240 (1) the non-uniform exposure of the FF seal elements to irradiation field and (2) the limited proton (3a) and electron (3d) exposure as compared to neutrons (3b) and $\gamma$-rays (3c). It is therefore anticipated that the effects on the ferrofluid and on the permanent magnet will be induced by these latter two irradiating species.
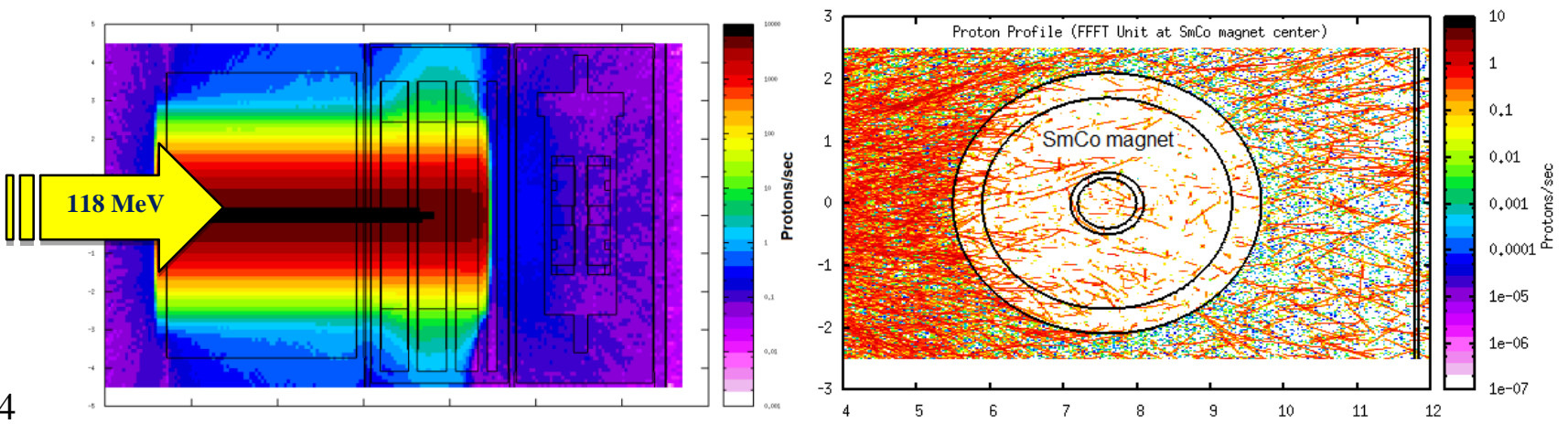

Figure 3a: Overall proton distribution profile (left) and over the mid-plane of FF (right) 

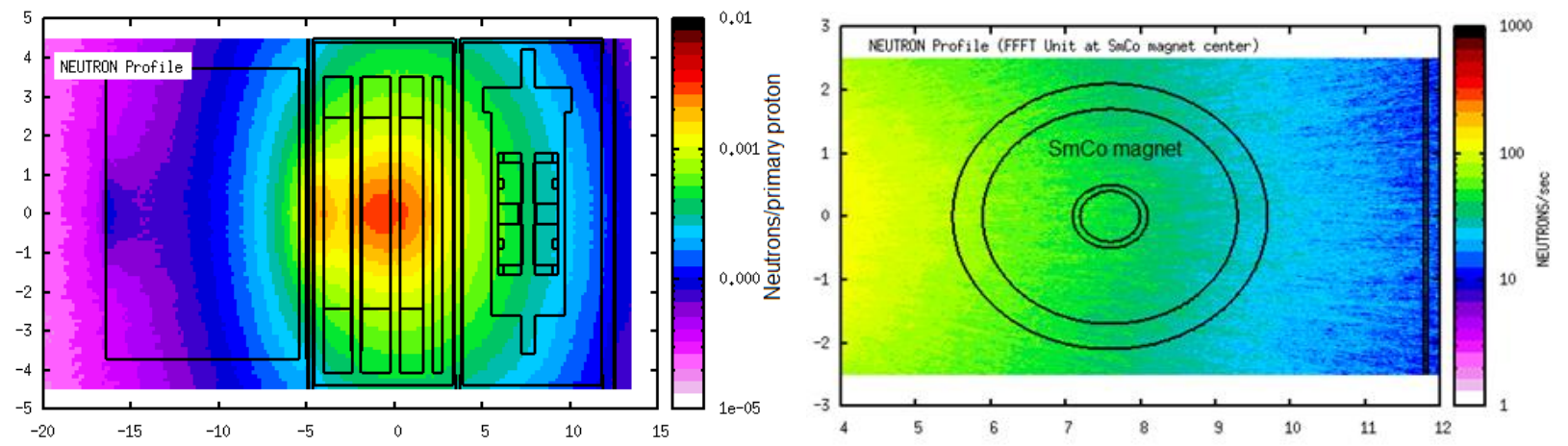

Figure 3b: Global neutron distribution profile (left) and aver the mid-plane of FF (right)
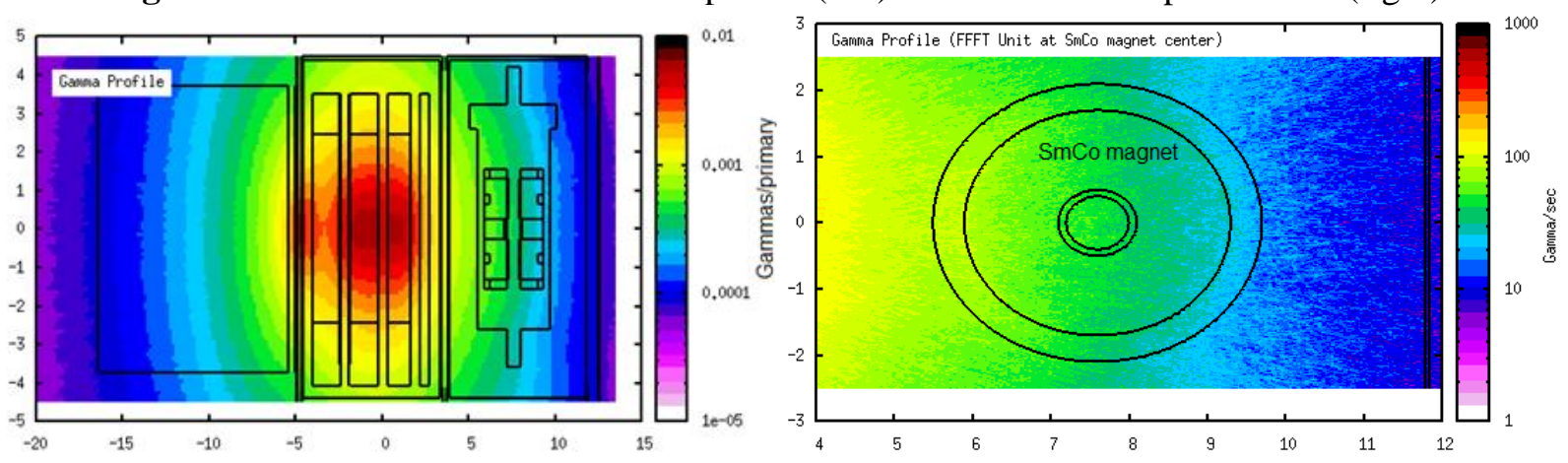

Figure 3c: Global $\gamma$-ray distribution profile (left) and aver the mid-plane of FF (right)
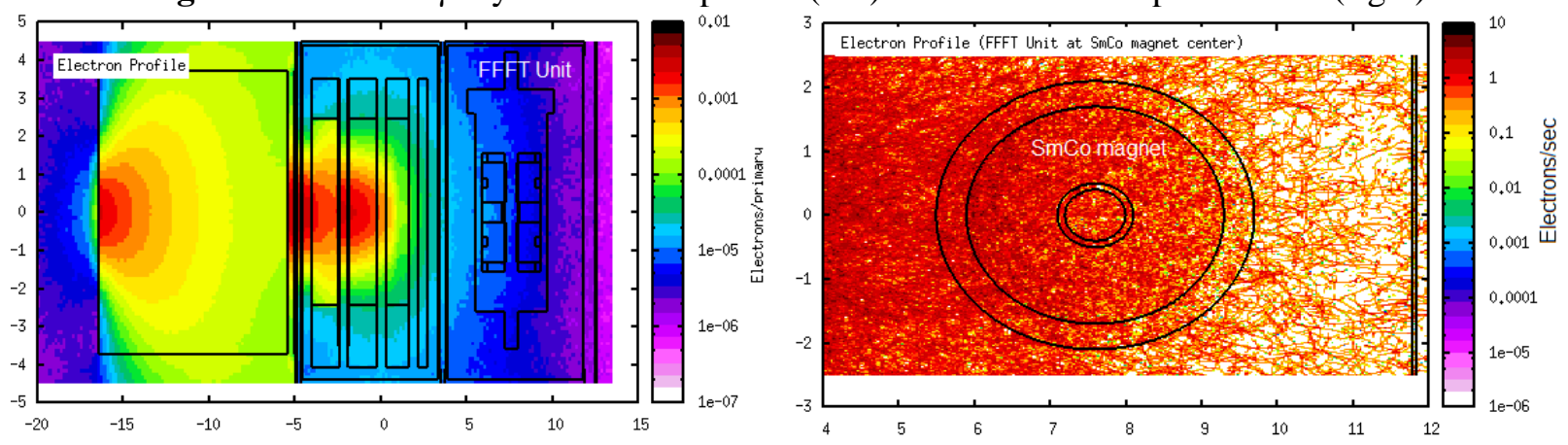

Figure 3d: Global electron distribution profile over the entire irradiation volume and FF seal (left) and over horizontal mid-plane cross section of FF seal (right) of the four irradiating species comprising the mixed irradiation field

As shown in Fig. 3b, which displays the profile of neutron intensity in the middle section of the Sm-Co magnet (parallel to the irradiating flux), the Sm-Co magnet is not irradiated uniformly, with peak fluences experienced on the upstream face of the annular magnet. The effect that such an irradiation profile would have on the demagnetization and the disturbance of the magnetic field is not known. Given that the magnet is responsible for the magnetic field that makes the ferrofluid function possible, it is reasonable to assume that any performance degradation of the FF seal is a collusive effect of the two primary systems, the magnet and the ferrofluid. Estimated dose profiles over the mid-section section of the FF seal exposed to 20 MGy are depicted in Figure 4.

For the $20 \mathrm{MGy}$ Sm-Co magnet and in particular for the location in the upstream face (Figure 2) the following important irradiation information was deduced regarding flux and fluence:

- Proton flux $1.2 \times 10^{8} \mathrm{p} / \mathrm{cm}^{2} / \mathrm{s}$ and proton peak fluence $1.14 \times 10^{14} \mathrm{p} / \mathrm{cm}^{2}$

- Neutron flux $8.6 \times 10^{10} \mathrm{n} / \mathrm{cm}^{2} / \mathrm{s}$ and peak neutron fluence $8.2 \times 10^{16}$ neutrons $/ \mathrm{cm}^{2}$ 
- $\gamma$ flux $1.31 \times 10^{11} \gamma / \mathrm{cm}^{2} / \mathrm{s}$ with peak $\gamma$ fluence $1.24 \times 10^{17} \gamma / \mathrm{cm}^{2}$

- Electron flux $2.85 \times 10^{9} \mathrm{e} / \mathrm{cm}^{2} / \mathrm{s}$ and electron fluence $2.7 \times 10^{15} \mathrm{e} / \mathrm{cm}^{2}$

These values, compared to experimental studies on listed in literature are typically about an order of magnitude higher except for electron-induced demagnetization reported [19] where a maximum fluence of $\sim 210^{15}$ electrons was used to study demagnetization of $\mathrm{Nd}_{2} \mathrm{Fe}_{14} \mathrm{~B}$ magnets.

At the ferrofluid location the flux and fluence of the irradiating species (neutrons, $\gamma$ and electrons) corresponding to the maximum dose of $20 \mathrm{MGy}$ are estimated to be of the order:

- Neutron flux $1.98 \times 10^{10} \mathrm{n} / \mathrm{cm}^{2} / \mathrm{s}$ and peak neutron fluence $1.88 \times 10^{16}$ neutrons $/ \mathrm{cm}^{2}$

- $\quad \gamma$ flux $2.57 \times 10^{10} \gamma / \mathrm{cm}^{2} / \mathrm{s}$ with peak $\gamma$ fluence $2.44 \times 10^{16} \gamma / \mathrm{cm}^{2}$

- Electron flux $3.81 \times 10^{8} \mathrm{e} / \mathrm{cm}^{2} / \mathrm{s}$ and electron fluence $3.62 \times 10^{14} \mathrm{e} / \mathrm{cm}^{2}$
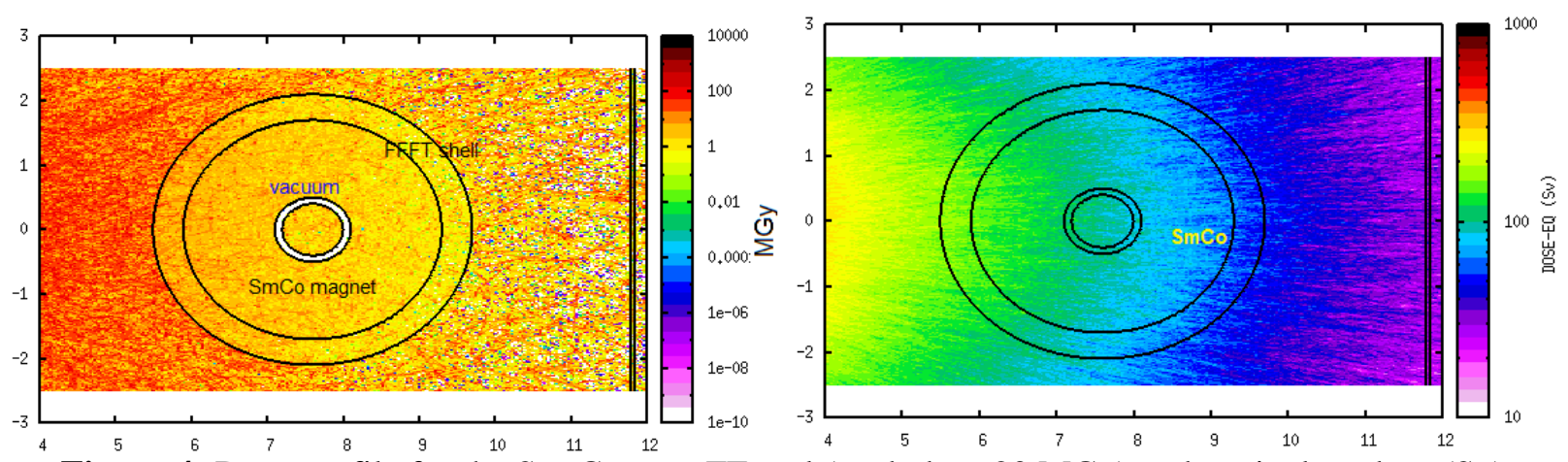

Figure 4: Dose profile for the Sm-Co-type FF seal (peak dose $20 \mathrm{MGy}$ ) and equivalent dose (Sv)
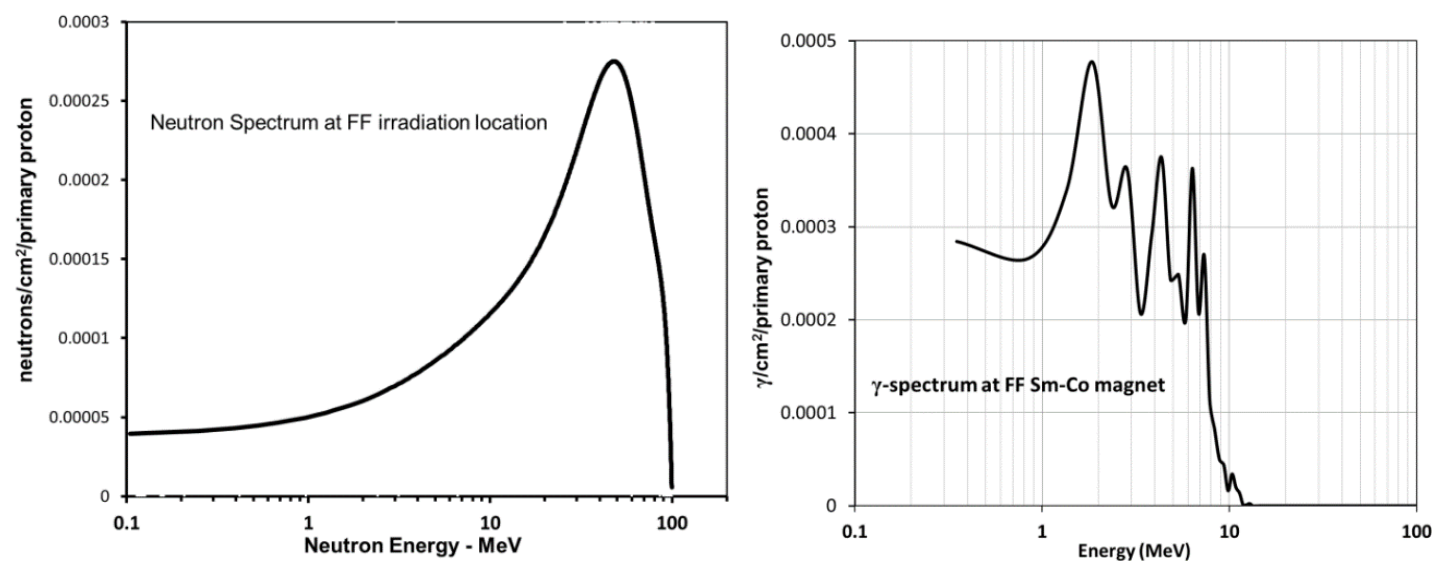

Figure 5: Neutron and $\gamma$ energy spectra at the upstream face of the Sm-Co magnet of the irradiated FF

Important information that can be extracted from Figure 5 is the spectral energies the different species (neutrons and $\gamma$-rays) have at the magnet location. Figure 5 shows that the neutron spectrum is dominated by faster (higher energy) neutrons. $\Gamma$-rays shower the upstream face of Sm-Co with energies up to 10 $\mathrm{MeV}$. These precise energy estimates, in combination with irradiation fluxes, particularly fluences, will help in correlating and, or comparing results deduced from other studies on radiation-induced demagnetization of permanent magnets.

\section{Post-irradiation Performance Assessment}

The post-irradiation performance assessment consisted of (a) measuring activation levels and photon spectra to correlate with dose levels (measured primary proton beam current in units of $\mu \mathrm{A}-\mathrm{hrs}$ and 
estimated fluxes of mixed field constituents), (b) integrated FF seal permanent magnet de-magnetization and (c) operational performance of the FF seal at conditions including rotational speeds reflective of anticipated operation at FRIB.

\subsection{Activation and Photon Spectra}

Upon completion of each of the three (3) FF seal irradiations $\gamma$-spectra were obtained using the ORTEC high-sensitivity Ge detector available at the Isotope Extraction Facility. The results of the three $\gamma$ spectra measurements made at EOB at are shown in Figure 6. A six-month long cool-down period for the three FF seals was allowed enabling the decay of radioactivity to below facility limits so performance testing of the FF seals in a HEPA filtered fume hood could be conducted.
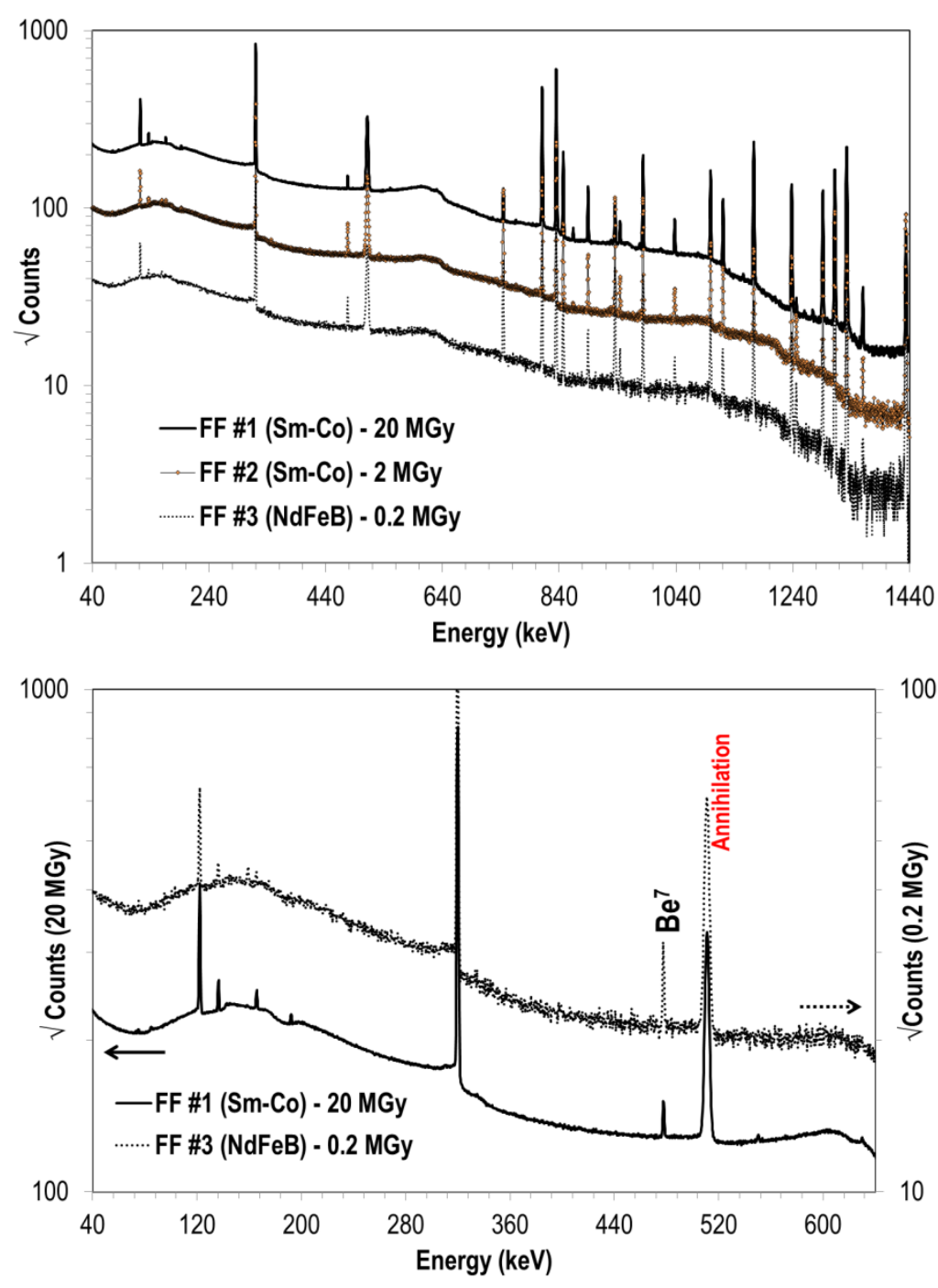

(a) 100

Figure 6: Photon spectra measured from the entire FF seal following irradiation.

The results of Figure $6 \mathrm{a}$ confirm the differences of irradiation doses achieved plus contain the subtle differences in the spectra between Units $1\left(\mathrm{Sm}_{2} \mathrm{Co}_{17}\right)$ with the spectra of Unit $3\left(\mathrm{Nd}_{2} \mathrm{Fe}_{14} \mathrm{~B}\right)$ while identifying the radioisotopic composition resulting from the entire $\mathrm{FF}$ seal. Isotopic photon lines attributed to the type of magnet $\left(\mathrm{Sm}_{2} \mathrm{Co}_{17}\right.$ or $\left.\mathrm{Nd}_{2} \mathrm{Fe}_{14} \mathrm{~B}\right)$ and the ferrofluid can be identified in the spectrum following a rigorous analysis. The $\mathrm{Nd}_{2} \mathrm{Fe}_{14} \mathrm{~B}$-based magnet $\mathrm{FF}$ seal was identified by comparing the spectra obtained following the irradiation of an $\mathrm{Nd}_{2} \mathrm{Fe}_{14} \mathrm{~B}$ permanent magnet [20] and in particular by 
focusing on the $\mathrm{Nd}^{147}$ and $\mathrm{Ce}^{139}$ isotopes with significant half-lives (11.06d and 137.6d respectively). Several reaction types are possible for the neutron energies involved and Neodymium including (n, $\gamma$ ), $(n, 2 n),(n, p),(n, p x n),(n, \alpha)$, and $(n, \alpha n)$ reactions. The spectra shown can also provide the benchmarking basis for the analyses that were performed using the transport codes, i.e. FLUKA, in terms of the isotopes that are predicted to be generated during irradiation via those observed in the activation analysis. Matching the isotopic photon lines and the activity, that are measured precisely by the detector, will provide confidence in the use of transport codes to establish the parameters of the irradiating mixed field at the FF components of interest.

\subsection{De-magnetization of Permanent Magnets - Radiation Effects on Annular Permanent Magnets $\left(\mathrm{Nd}_{2} \mathrm{Fe}_{14} \mathrm{~B}\right.$ and $\left.\mathrm{Sm}_{2} \mathrm{CO}_{27}\right)$}

Limited experience is available on the radiation-induced degradation of ferrofluids from energetic protons, thermal and fast neutrons, rays and electrons. Thus the effects of radiation on the chemical and physical properties of ferrofluids are not yet well understood and the studies are scarce. Yoshida and coworkers [7], as part of the radioactive-isotope beam separator project (BigRIPS), studied the radiation hardness of the magnetic fluid in vacuum-tight actuators. They have studied changes in the viscosity, surface tension and colloid stability of ester-based ferrofluid by irradiation with intense $\gamma$ rays. They reported that no significant changes of the viscosity were observed for doses in the range of 0.7-1.8 MGy.

More relevant to the present study, an irradiation experiment was also conducted by Yoshida and coworkers [7] where the performance of a rotating actuator placed in the radiation field of high-energy neutrons and light charged particles was assessed. For an accumulated dose of $0.2 \mathrm{MGy}$ and periodic testing of the rotational property of the actuator, no vacuum leak was observed. In the present study, accumulated doses of 0.2MGy, 2.0 MGy and $20 \mathrm{MGy}$ have been reached for the three FF seals from a mixed spectrum. The two irradiated states and the performance of the present FF seals can be compared directly with the results of [7]. Further evidence of the irradiation effect on ferrofluids was offered in [12] where magnetization of kerosene-based ferrofluid containing $\mathrm{Fe}_{3} \mathrm{O}_{4}$ has been studied. Influence on agglomeration and sedimentation in the ferrofluid was observed with doses up to only $4.5 \mathrm{~Gy}$.

In the present study, using a Hall probe, magnetic field strength measurements were conducted over an array of locations (Figure 7). The pre-irradiation and post-irradiation measurements (latter conducted remotely) were not meant to be exact, given the inaccessibility of the surface of the magnets themselves, but rather to compare the effects of dose on the relative changes of the magnetic field intensity by comparing the response of the $\mathrm{Nd}_{2} \mathrm{Fe}_{14} \mathrm{~B}$ magnets with the $\mathrm{Sm}_{2} \mathrm{Co}_{27}$ counterparts. Similar magnetic field intensities were measured for the as-received (unirradiated) $\mathrm{Sm}_{2} \mathrm{Co}_{17}$ and $\mathrm{Nd}_{2} \mathrm{Fe}_{14} \mathrm{~B}$-based $\mathrm{FF}$ seals.

By utilizing a similar Hall probe set-up within the facility hot cell, and via remote operation, scanning of the three (3) irradiated FF seals (0.2 MGy for the NdFeB-based FF seal and 2 MGy and 20 MGy for the SmCo-based FF seals) was performed. Figure 8 compares measured magnetic field intensity data for asreceived and irradiated FF seals. It should be noted that effort was made to conduct measurements of the magnetic fluxes with similar accuracy for both irradiated and unirradiated units. The measurements were made with the Hall probe $\sim 2 \mathrm{~mm}$ above the surface of the FF seal with the $2 \mathrm{~mm}$ thick non-magnetic probe's base support in contact with the FF seal surface. Due to the inaccessibility of the encased permanent magnet and the remote operation in measuring the residual field of the irradiated units (through the hot cell wall) the measurements can only be considered as qualitative. The data show a striking similarity of the magnetic field intensity variation despite the wide range of dose. The findings indicate that minimal, if any at all, de-magnetization was experienced by both types of magnets integrated into the FF seals. 
Figure 7: Magnetic field measuring locations and Hall probe application.
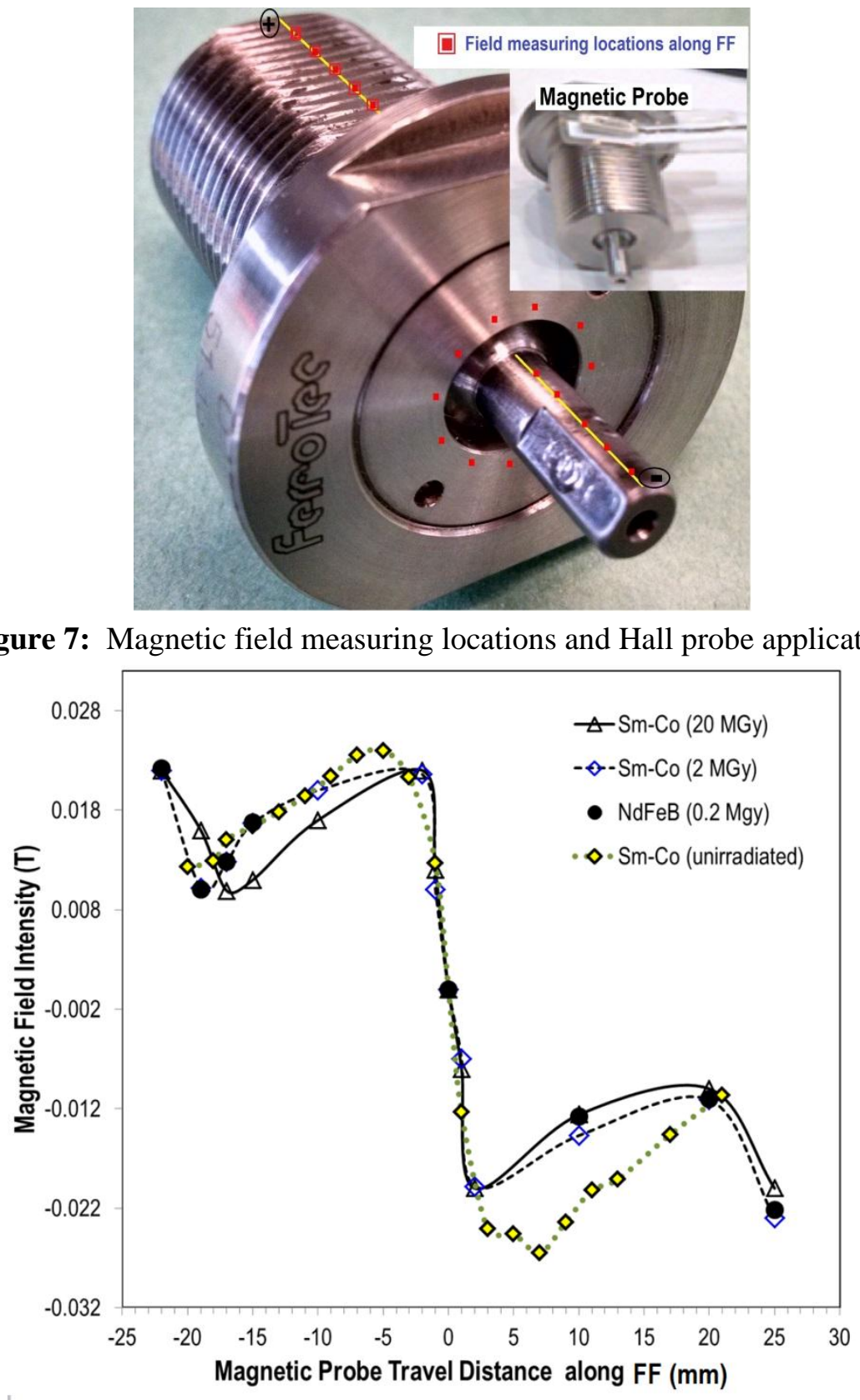

Figure 8: Measured magnetic field intensity versus magnetic probe travel along the flattened shaft of the irradiated FF seals shown in Fig. 7. Position $0 \mathrm{~mm}$ defines the magnet center plane.

372 Experimental studies of the de-magnetization of permanent magnets of the types explored here have been conducted. Specifically, Zeller [16] studied $\mathrm{Nd}_{2} \mathrm{Fe}_{14} \mathrm{~B}$ with $\gamma$-rays from a ${ }^{60} \mathrm{Co}$ source and $106 \mathrm{MeV}{ }^{2} \mathrm{H}$ ions and reported that while the de-magnetization effect of the $\gamma$-rays was only a few percent, over $80 \%$ of flux loss was measured at 2.6 MRad. Kankonen et al [17] irradiated $\mathrm{Nd}_{2} \mathrm{Fe}_{14} \mathrm{~B}$ with protons up to 1000 MRad and found that loss to be strongly dependent on both the irradiation temperature as well as shape of the sample. Specifically, plate-shape samples magnetized perpendicular to the surface were most sensitive to radiation and experienced severe flux loss while samples with magnetic lines parallel to the larger surface exhibited remarkable de-magnetization resistance. This behavior is attributed $[\mathbf{1 6}, \mathbf{1 8}]$ to the fact that the demagnetizing forces are small when the magnet is magnetized parallel to the large dimension 
and thus less sensitive to irradiation. In the case of an annular magnet (as is the case of FF with NdFeB or SmCo annular magnets) that is magnetized parallel to the thin dimension, a large portion of the demagnetization is removed by removing the low permeance central part where the demagnetization forces are larger. As a result, the annular $\mathrm{NdFeB}$ permanent magnet with a large part of its demagnetizing force removed exhibits insensitivity to irradiation.

$\mathrm{Nd}_{2} \mathrm{Fe}_{14} \mathrm{~B}$ de-magnetization studies [20] from a similar mixed field irradiation and for dose in the range of 50-1800 MRad showed that severe demagnetization (>90\%) occurs even at 50 MRad levels as seen in Figure 9. The neutron fluence levels for the $\mathrm{Nd}_{2} \mathrm{Fe}_{14} \mathrm{~B}$ magnets studied in [20] were in the range of $1.29 \times 10^{16} \mathrm{n} / \mathrm{cm}^{2}-4.38 \times 10^{17} \mathrm{n} / \mathrm{cm}^{2}$ (peak dose of $1.8 \mathrm{Grad}$ or $18 \mathrm{MGy}$ ).

Regarding the de-magnetization resilience of the $\mathrm{Sm}-\mathrm{Co}$ magnets as compared to NdFeB magnets, irradiation studies using fast neutron fluences of $4 \times 10^{12} \mathrm{n} / \mathrm{cm}^{2}[\mathbf{1 8}]$ at $350 \mathrm{~K}$ showed that the magnetic field intensity decay rate of $\mathrm{Nd}-\mathrm{Fe}-\mathrm{B}$ magnets was significantly faster than the rate of its Sm-Co counterpart. Similar conclusions were reached in [19] using electron fluxes where it was revealed [19] that Sm-Co magnets exhibited much high resistance to magnetic field intensity reduction $\left(\sim 1.4 \%\right.$ reduction in $\mathrm{SmCo}_{5}$ after exposure to $4 \times 10^{14}$ electrons as compared to $\sim 10 \%$ reduction in $\mathrm{Nd}_{2} \mathrm{Fe}_{14} \mathrm{~B}$ for the same exposure at $300 \mathrm{~K})$.

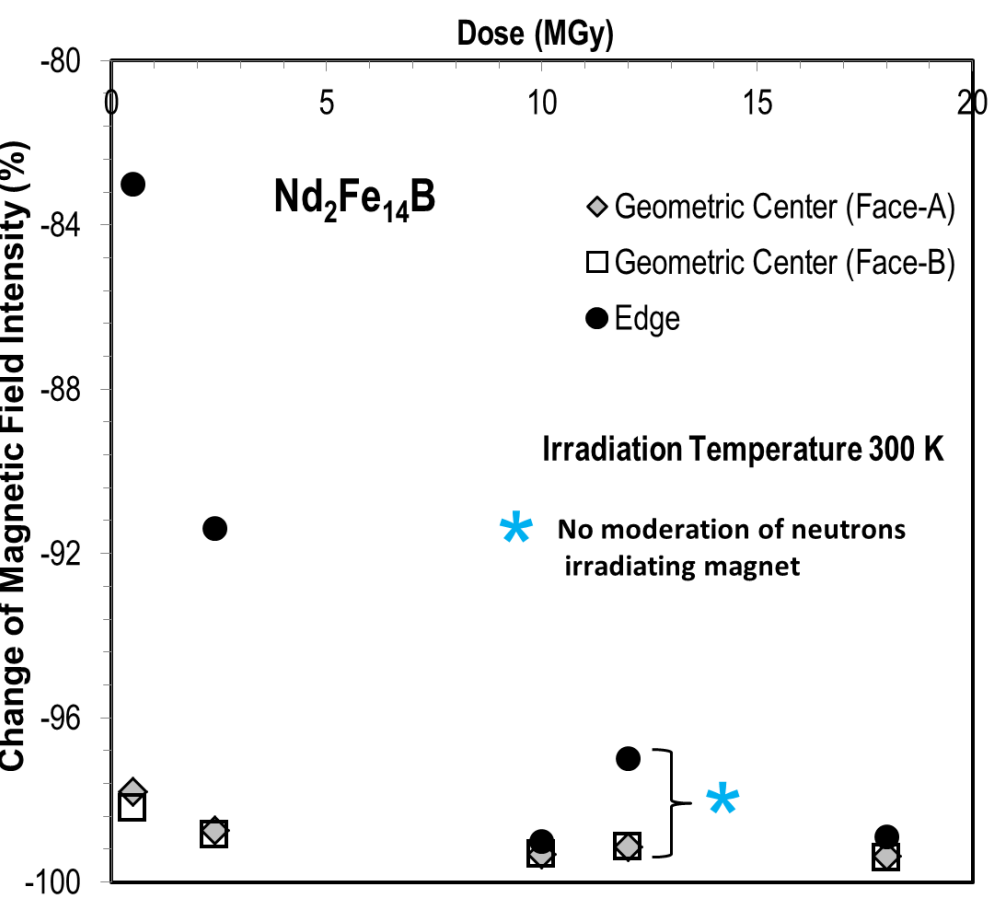

Figure 9: De-magnetization of $\mathrm{Nd}_{2} \mathrm{Fe}_{14} \mathrm{~B}$ rectangular magnets $(5 \mathrm{~cm} \times 4.75 \mathrm{~cm} \mathrm{x} 0.7 \mathrm{~cm})$ magnetized through thickness from mixed field irradiation [20]

At the $0.2 \mathrm{MGy}$ dose level for the $\mathrm{Nd}_{2} \mathrm{Fe}_{14} \mathrm{~B}$-based $\mathrm{FF}$ seal and given the de-magnetization experience [20] it should have been expected that the permanent magnet would undergo substantial de-magnetization ( $\sim 95 \%$ reduction at the magnet center for $0.5 \mathrm{MGy}$ dose was observed [20] for $\mathrm{Nd}_{2} \mathrm{Fe}_{14} \mathrm{~B}$ magnets in a similar irradiating field, see Figure 9). As discussed above, the annular magnet shape where the part with the strongest demagnetizing forces is removed, greatly influences the degree of de-magnetization due to irradiation. 


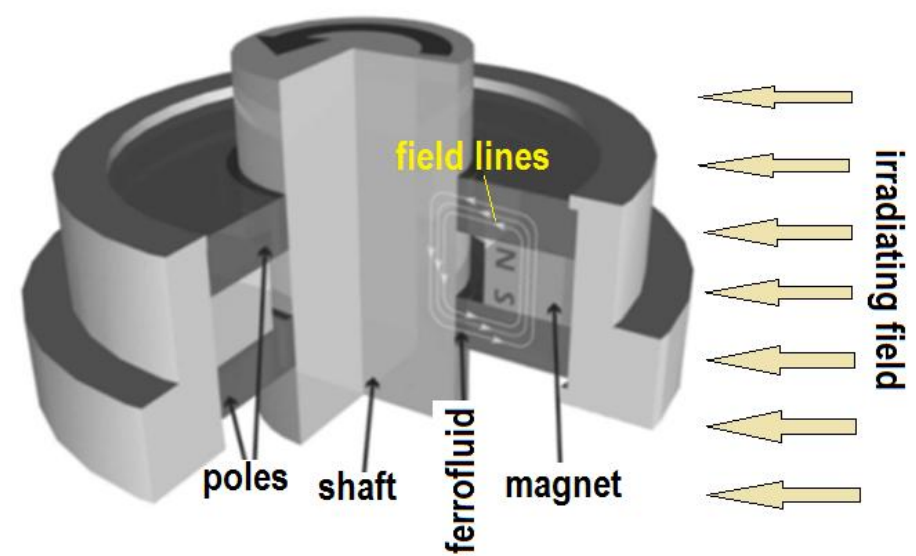

Figure 10: Magnetization orientation in ferrofluidic feedthroughs vs. irradiation direction

\subsection{Ferrofluid Feedthrough Post-irradiation Performance}

The post-irradiation FF performance was evaluated based on the static torque, the breakaway torque, the rotational torque and the leak rate. The ferrofluid in the FF and the rotating shaft ball bearings inherently present a certain resistance to rotation due to the frictional forces which result from the actuation of viscous and pressure drag forces in the ferrofluid, and due to the friction occurring at all surfaces of contact with the FF shaft. Assuming that the system friction is negligible compared to the viscous forces developed in the ferrofluid, the static torque can be estimated from the shaft rotation induced under specified conditions. The static torque $T_{s t}$ can be expressed as a function of the inverse of $t_{d}$, $t_{d}$ being the time required, following the initiation of the feedthrough rotation, to reach a specified displacement distance $d$ under a gradually increasing imbalance of counterweights acting on the FF shaft via a timing belt with the two counterweights at each end. An integrated test system that is depicted and described in Figure 11 was used in the radiation area to study the post-irradiation evaluation.
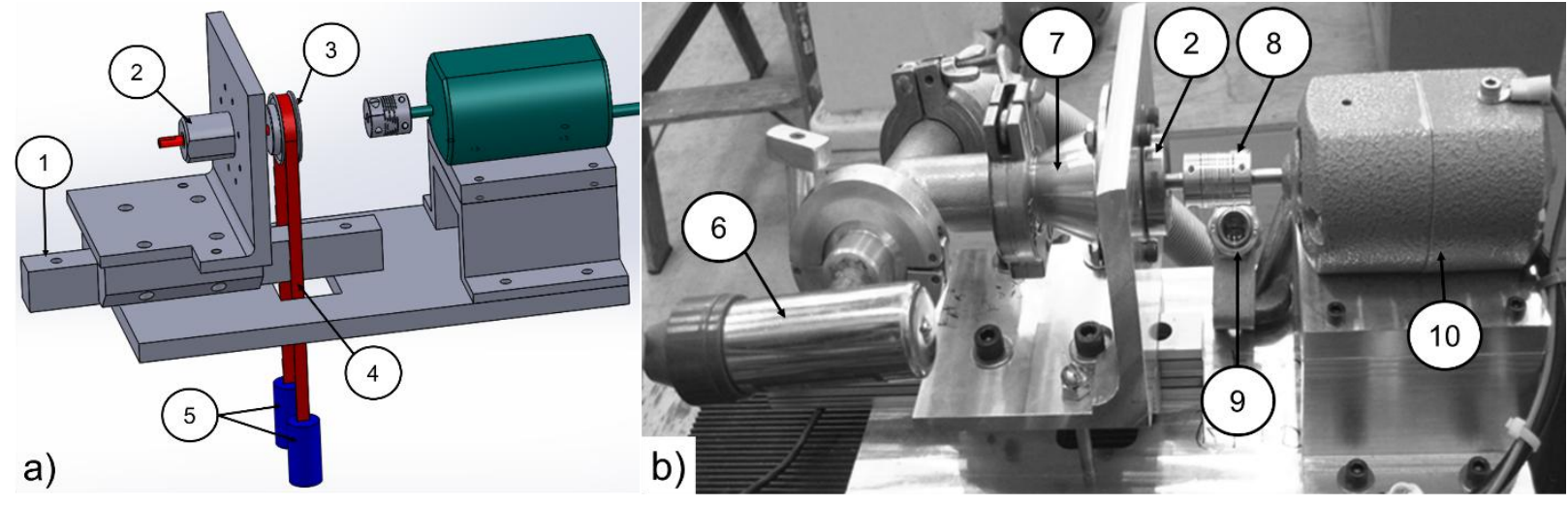

Figure 11: Torque testing set-up: (a) schematic of static torque testing (b) photograph of rotational torque testing set-up. Legends: (1) linear transmission guide, (2) ferrofluidic feedthrough, (3) gear belt pulley, 4) timing belt, (5) counter-weights of mass $\boldsymbol{m}_{\boldsymbol{l}}$ and $\boldsymbol{m}_{2}$, (6) primary pump gauge, (7) cone with FF connected to the vacuum system, (8) flexible shaft coupling, (9) digital tachometer, and (10) AC/DC motor.

The static torque was measured following a rotational cycle of the system at rotational speeds up to 5,000 $\mathrm{rpm}$, which helped activate the magneto-viscous conditions in the ferrofluid and while the ferrofluid was expected to be at elevated temperatures. Static torque measurements were made on the non-irradiated FF 
434

(as a baseline) and on the FF seals that experienced doses of 0.2 and 2 MGy. Ferrofluid leakage detected prior to any post-irradiation testing in the highest dosed FF (20 MGy) indicating radiation damage to the ferrofluid (excluding any contribution from the de-magnetization of the permanent magnet as shown in Section 3.2) prevented any experimental data on static and rotational torque to be deduced. Figure 12 depicts the measured static torque as a function of the inverse of displacement time $\left(1 / t_{d}\right)$. Systematic errors stemming from combined inaccuracies in measuring the resulting displacement distance $d$ (kept constant for all tests at $20 \pm 0.5 \mathrm{~cm}), 1 / t_{d}$ and acquisition time were estimated to be of the order of $14 \%$.

In addition to the static torque, the initial force required for starting the FF rotational motion from rest, known as the breakaway torque, is of interest. The breakaway torque can be estimated by extrapolating the static torque $T_{s t}$ trend when expressed as a function of the inverse of $t_{d}$ for $1 / t_{d} \rightarrow 0$.

Similar breakaway torque values were observed for as-received, unirradiated $\mathrm{Nd}_{2} \mathrm{Fe}_{14} \mathrm{~B}$-based and $\mathrm{Sm}_{2} \mathrm{Co}_{27}$-based FFs. The values of breakaway torque for the different FFs that were tested are summarized in Table 2 and vary between 2 - $17 \mathrm{~N}-\mathrm{mm}$.

Lower breakaway torque values were obtained for the tests where the FF has previously undergone rotational speed cycles. Significant reduction in breakaway torque was observed even when the FF was subjected to manual rotation prior to the start of the test. To further quantify the history-dependence of the behavior of the breakaway torque on the change of the ferrofluid physical properties the FF irradiated to 0.2 MGy was subjected to a sequence of rotational cycles while monitoring the evolution of the breakaway torque with each cycle. Given the inherent inability to monitor the temperature evolution of the ferrofluid during a rotational cycle, a 20-minute cool-down period was introduced before a new rotational cycle began thus allowing the ferrofluid to return to room temperature.

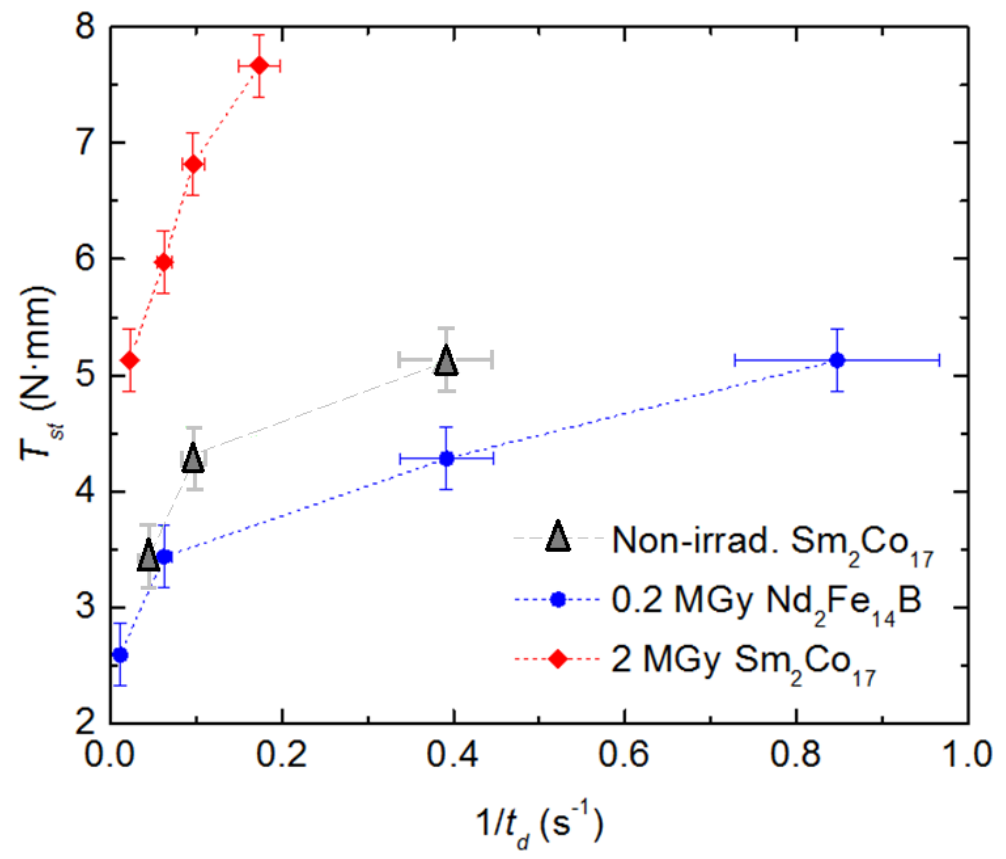

Figure 12: FF static torque $\boldsymbol{T}_{\boldsymbol{s t}}$ vs. the inverse of displacement time $\left(1 / t_{d}\right)$ following rotational speed cycles up to 5,000 rpm.

The rotational tests indicated that an initial breakaway torque of $17 \pm 3 \mathrm{~N}$-mm decreased to $7 \pm 2 \mathrm{~N}$-mm and $5 \pm 0.5 \mathrm{~N}$-mm following rotational cycles to 5,000 rpm. Subsequently, and following a third rotational cycle to $5,000 \mathrm{rpm}$, the $0.2 \mathrm{MGy}$ dose FF breakaway torque approached the non-irradiated value. Table 2 depicts the breakaway torque results from the array of tests. No significant variation in the 
464

465

466

467

468

469

470

471

472

473

474

475

476

477

478

479

480

481

482

483

484

485

486

487

488

489

490

491

492

493

494

495

496

497

498

499

breakaway torque is observed immediately after a rotational speed cycle for the non-irradiated FF and the FF at 0.2 MGy. The FF irradiated with $2 \mathrm{MGy}$ exhibited a breakaway torque of $4.7 \pm 0.5 \mathrm{~N}-\mathrm{mm}$ immediately after the first rotational cycle of 5,000 rpm, a $70 \%$ increase compared to the unirradiated FF of the same magnet type (Sm-Co) and under identical measurement conditions.

While the breakaway torque cannot be used as reliable measure for comparison between different feedthroughs, especially when the ferrofluid has been at prolonged static conditions, is at temperature below the optimal, and uncertainties exist regarding the mixing/processing history and ferrofluid colloidal suspension stability, it is an indicator of disorder in the ferrofluid which can be further accentuated by radiation damage manifested in changes in the ferrofluid physical properties. As the tests indicate, minima in breakaway torque are reached when stabilization effects of the ferrofluid colloidal suspension are attained via solid-body rotation [14] or once the viscosity decrease is realized due to temperature increase. High initial starting torque is attributed to sedimentation or aggregation of the ferrofluid colloidal particles [12] resulting from prolonged rest state and (b) sedimentation, aggregation and viscosity degradation resulting from the irradiation. As a case in point, the static torque for the $20 \mathrm{MGy}$ FF seal was not measured due to the fact that its shaft was practically locked and immobilized. The necessary torque to initiate rotation of the irreparably damaged $20 \mathrm{MGy}$ FF that exhibited ferrofluid leakage due to the irradiation was manually applied and was in excess of 20,000 N-mm.

\section{$\underline{\text { Rotational torque }}$}

The rotational torque of each FF seal under dynamic conditions, $T_{r o t}$, was determined by using the testing set-up with the addition of a primary vacuum pump system enabling one side of the FF to operate under vacuum during the tests, a digital tachometer for rotational speed measurement and an AC/DC motor for inducing rotation of the FF. The instantaneous alternating current and voltage drop across the motor, as a function of the increasing and decreasing rotational speed were monitored while the motor was either decoupled or coupled to the FF seal through a flexible helical beam coupling. The FF rotational torque is computed as the difference between the total rotational torque $T_{\text {rot }}=P / \omega(\mathrm{N}-\mathrm{m})$ and the rotational torque of the decoupled motor. $P$ represents the electrical power $(\mathrm{W})$ and $\omega$ the angular velocity $\left(\mathrm{rad} \cdot \mathrm{s}^{-1}\right)$. Figure 13 depicts the FF rotational torque for the unirradiated and the irradiated FFs for the two lower doses (0.2 and 2 MGy). As seen in Figure 13, a similar level torque was required to start-up the 0.2 MGy irradiated FF and the non-irradiated FF. No change on the rotational torque of the feedthroughs irradiated to 0.2 and 2 MGy was observed in comparison to a non-irradiated FF as the rotational speed increases leveling off at 5,000 rpm.

Table 2: Measured FF breakaway torque values for different irradiation conditions

\begin{tabular}{|c|c|c|c|}
\hline $\begin{array}{c}\text { Magnet } \\
\text { composition }\end{array}$ & $\begin{array}{c}\text { Dose } \\
\text { (MGy) }\end{array}$ & $\begin{array}{c}\text { Breakaway torque } \\
(\mathbf{N}-\mathbf{m m})\end{array}$ & Experimental conditions \\
\hline \multirow{2}{*}{$\mathrm{Sm}_{2} \mathrm{Co}_{17}$} & \multirow{2}{*}{0} & $4 \pm 0.4$ & Non-irradiated \\
\hline & & $3 \pm 0.3$ & following a rotational speed cycle \\
\hline \multirow{4}{*}{$\mathrm{Nd}_{2} \mathrm{Fe}_{14} \mathrm{~B}$} & \multirow{4}{*}{0.2} & $17 \pm 3$ & Post- irradiation, no previous rotation \\
\hline & & $7 \pm 2$ & After $1^{\text {st }}$ rotational speed cycle \\
\hline & & $5 \pm 0.5$ & After $2^{\text {nd }}$ rotational speed cycle \\
\hline & & $2.5 \pm 0.2$ & After $3^{\text {rd }}$ rotational speed cycle \\
\hline $\mathrm{Sm}_{2} \mathrm{Co}_{17}$ & 2.0 & $5 \pm 0.5$ & After $1^{\text {st }}$ rotational speed cycle \\
\hline
\end{tabular}




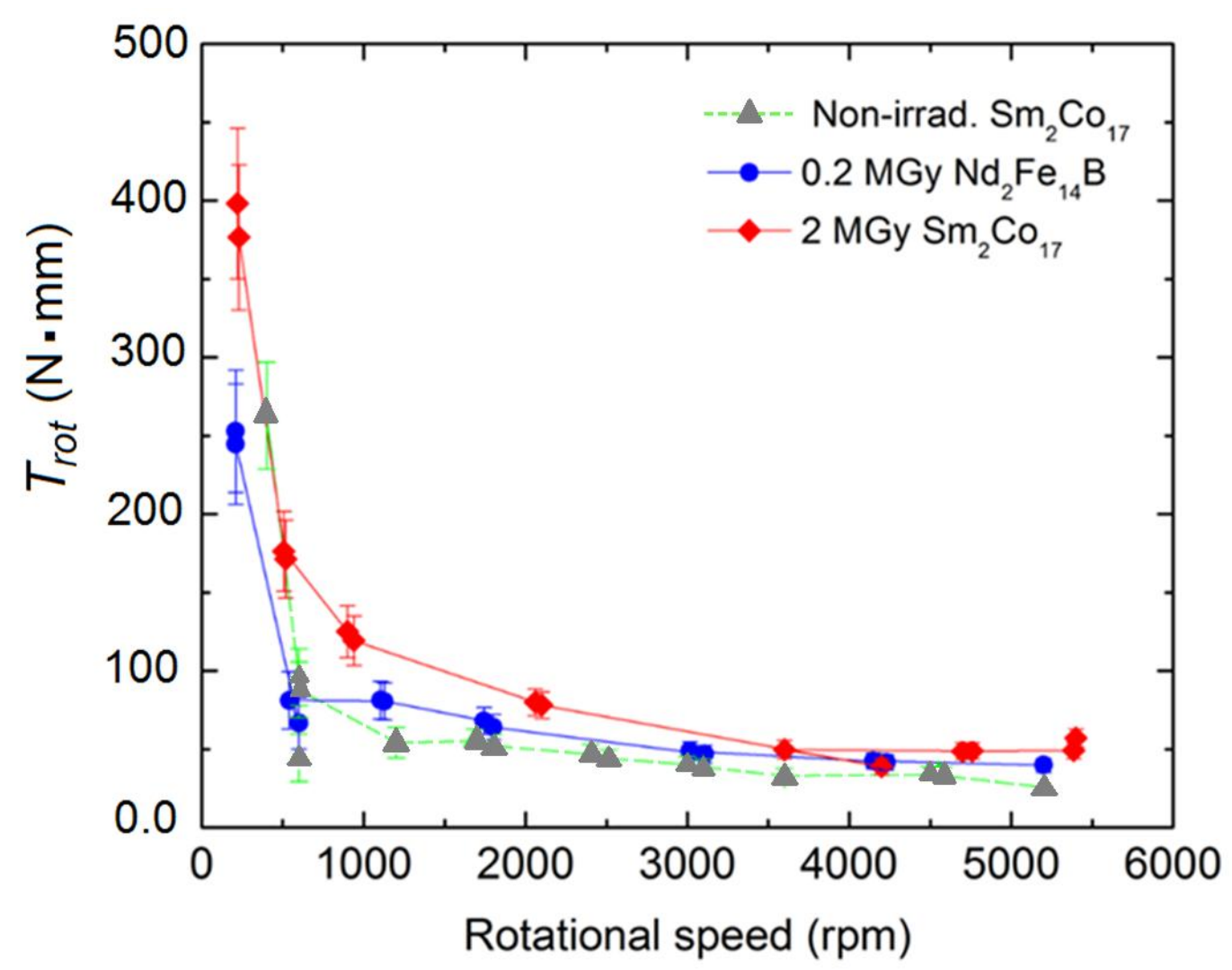

Figure 13: FF rotational torque vs. the rotational speed as a function of radiation dose.

The results of a study [14] where the relationship between the magnetization and magnetic field in a ferrofluid rotating like a rigid body was studied experimentally and theoretically are brought into focus to explain the observed behavior or restoration of the "rotational viscosity" following solid-body rotation of the ferrofluid to 5,000 rpm in the present study. The magneto-viscous effect and consequently the rotational dynamics of the nanoscaled magnetic particles in a ferrofluid are influenced by imposed macroscopic flow and/or by the application of a magnetic field. Experimental and theoretical results [14] for induced magnetization in the ferrofluid (or magnetic field change measured by a Hall sensor outside the ferrofluid cylinder) as a function of rotational speed confirmed the findings of the current study. Specifically, the maximum field measured by the sensor in [14] was shown to occur at rotational speeds of $500 \mathrm{rad} / \mathrm{s}$ which are close to the 5,000 rpm $(\sim 523 \mathrm{rad} / \mathrm{s})$ observed in the current study experiments on irradiated FF seals as shown in Fig. 13 where rotational speeds approaching 5,000 rpm restored the viscosity of the ferrofluid.

\section{Irradiation-induced Ferrofluid Feedthrough Seal Leak}

A FF with a standard ferrofluidic seal can operate in high-vacuum systems $\left(1 \times 10^{-7} \mathrm{~Pa}\right)$ with leak rates below $10^{-11}$ standard $\mathrm{cm}^{3} \mathrm{He} \cdot \mathrm{s}^{-1}[6]$. Following the irradiation of the three (3) FF seals while being cognizant of the potential of contaminating the high-precision helium leak detector, equipment leak rates under both static and dynamic conditions for up to 5,000 rpm were deduced from measurements of the outgassing rate that results from the experimental set-up and the ferrofluid seal leakage. Figure 14 depicts the post-irradiation and post-dynamic test state of the two Sm-Co FFs. There are clear signs of ferrofluid leakage at the shaft coupling, indicative of the radiation-induced damage. To extract the ferrofluid seal leakage, the outgassing from the test set-up leak rate was estimated first under static and then dynamic conditions up to $5,000 \mathrm{rpm}$ by measuring the rate of pressure increase as a function of time after closing 
the valve between the vacuum system and the testing set-up. No leak rate differences were observed in the primary vacuum pumping system of the rotational torque testing set-up with or without the presence of a connected non-irradiated FF.
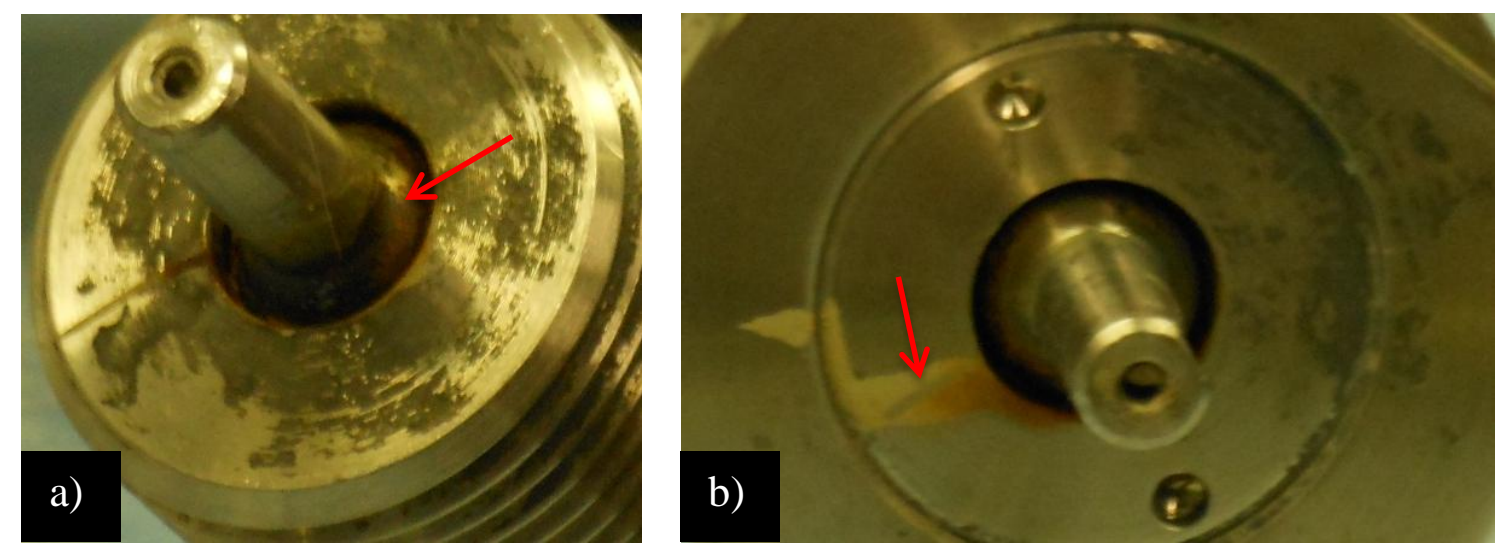

Figure 14: Evidence of ferrofluid leakage following radiation doses of (a) 2 MGy, (b) 20 MGy

The average rate for all the FFs under static and dynamic conditions was determined from the slope of the measured pressure as a function of time. The average leak rates computed are presented in Table 3 .

Table 3: Average outgassing rates extracted from the slopes of the linear fits of the experimental data. *Manually forced rotation of FF (20 MGy dose)

\begin{tabular}{|c|c|c|}
\hline \multirow{2}{*}{ FF reference } & $\begin{array}{c}\text { Rotational speed } \\
(\mathbf{r p m})\end{array}$ & $\begin{array}{c}\text { Average leak rate } \\
\boldsymbol{Q}\left(\mathbf{1 0}^{-\mathbf{3}} \mathbf{P a} \cdot \mathbf{L} \cdot \mathbf{s}^{-\mathbf{1}}\right)\end{array}$ \\
\hline \multirow{2}{*}{$\begin{array}{c}\text { Non-irradiated } \\
\left(\mathrm{Sm}_{2} \mathrm{Co}_{17}\right)\end{array}$} & 0 & $30.4 \pm 0.3$ \\
\cline { 2 - 3 } $0.2 \mathrm{MGy} \mathrm{Nd}$ & $3900-4200$ & $29.5 \pm 0.3$ \\
\hline \multirow{2}{*}{$2 \mathrm{FG}_{14} \mathrm{~B}$} & 0 & $23.0 \pm 0.3$ \\
\cline { 2 - 3 } $\mathrm{Sm}_{2} \mathrm{Co}_{17}$ & $3600-4430$ & $22.6 \pm 0.3$ \\
\cline { 2 - 3 } & 0 & $27.6 \pm 0.4$ \\
\hline \multirow{2}{*}{$20 \mathrm{MGy} \mathrm{Sm}_{2} \mathrm{Co}_{17}$} & $3600-5100$ & $37.7 \pm 0.6$ \\
\cline { 2 - 3 } & 0 & $25.6 \pm 1.0$ \\
\hline
\end{tabular}

Specifically, the average outgassing rate in the vacuum system for FFs not affected by radiation (including FFs irradiated up to $0.2 \mathrm{MGy}$ ) under static and dynamic conditions was estimated to be $\sim 23 \times 10^{-3} \mathrm{~Pa} \cdot \mathrm{L} \cdot \mathrm{s}^{-1}$. For the FF seal irradiated to $2 \mathrm{MGy}$ the relative total outgassing rate increased by $37 \%$ from static to dynamic conditions of 5,000 rpm. Hence, we can estimate that the FF gaseous leak rate is less or equal to $15 \times 10^{-3} \mathrm{~Pa} \cdot \mathrm{L} \cdot \mathrm{s}^{-1}$. This gaseous leak may be associated to radiation-induced degradation of the ferrofluid and/or of the static O-rings. For the FF seal irradiated to $20 \mathrm{MGy}$ a higher outgassing rate under static conditions was observed after manually forcing the FF rotation.

In an effort to assess and pinpoint the true source of the FF outgassing, gaseous products were collected during the dynamic test of the $2 \mathrm{MGy} \mathrm{Sm}-\mathrm{Co} \mathrm{FF}$. These collected products were studied using a high sensitivity Ge photon detector to extract isotopic photon signatures that can be linked to the FF seal and in particular the irradiated and damaged ferrofluid. Due to the anticipated weak signals from the outgassing 
volume, two photon signatures were deduced from the data collected by the Ge detector, one of which was obtained following purging of the detector to eliminate the possibility of cross-contamination from medical isotopes studied with the detector. The photon spectra of both measurements were identical and isotopic signature photopeaks were clearly identified and correlated to the photon spectra of the irradiated FF (regions of the spectra are shown in Figure 15). Arrows indicate matching isotopic signatures in the spectra of the irradiated FF seal and the outgas products. Fig. 15a depicts the comparison of the irradiated FF photon spectra (20 MGy and 0.2 MGy) in the energy region 20-240 keV. Figures 15(b-c) show excerpts of photon data with signatures of isotopes present in the outgassing products collected during the rotational tests of the $2 \mathrm{MGy}$ dose (Sm-Co) FF.

In addressing the performance of a $\mathrm{FF}$ in a radiation environment and in particular the role of individual components being affected by the onset of radiation dose (i.e. ferrofluid, permanent magnet and O-rings) while encased in a stainless steel shell, challenges in separating the potential degradation due to irradiation exist. This is further compounded by the proprietary nature of the composition of the components and in particular of the ferrofluid. While the degradation of the magnetic field can be assessed with a Hall sensor outside the volume, analysis of outgas or leak products and in particular their relative isotopic composition is the reliable approach for an in-situ operational performance assessment. To enable a predictive model that will account for (a) the exact irradiating field and (b) the volumetric and elemental composition of the FF and (c) correlate radionuclides observed with the composition of critical elements, a high fidelity Monte Carlo-based model was generated using the FLUKA transport code. Using the irradiation and decay time histories of the experiments, the photon spectra deduced for the FF seals as a whole and the photon signatures of the outgassing products, and the produced radionuclides were predicted by FLUKA and compared with the experimentally obtained data.

While the proprietary nature of the ferrofluid and thus the knowledge of the exact elemental composition prevent the one-to-one comparison and thus benchmarking, still a generic description of the ferrofluid implemented in the FLUKA predictive model offered a reliable corroboration of the anticipated isotopes with those observed experimentally, especially isotopes expected to be present in the activated ferrofluid. The objective of the benchmarking effort is to develop a reliable predictive model that will be used, in correlation with measured in-situ outgas isotopic profiles of ferrofluids in harsh irradiating fields, to predict the operational life expectancy of the FF seals as a whole. Figure 16 depicts excerpt results of the predictive model namely the $\gamma$ spectrum and isotopic composition. Arrows indicate selected photon signatures that are also recorded by the actual photon analysis measurements of the outgas products shown in Figure 15. The agreement between the predicted and measured spectra for the appropriate $\gamma$ energies originating from the anticipated isotopes generated in the ferrofluid provides confidence towards utilizing the developed model for in-situ assessment of FF leakage. 


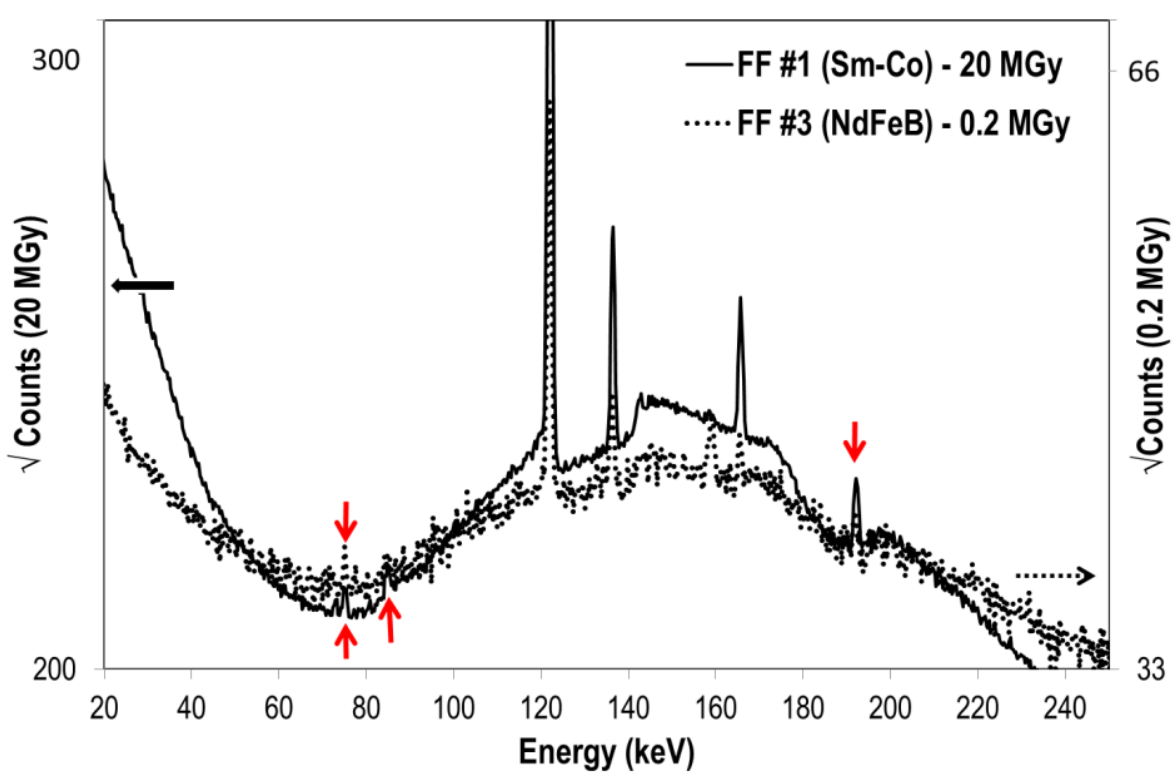

(a)

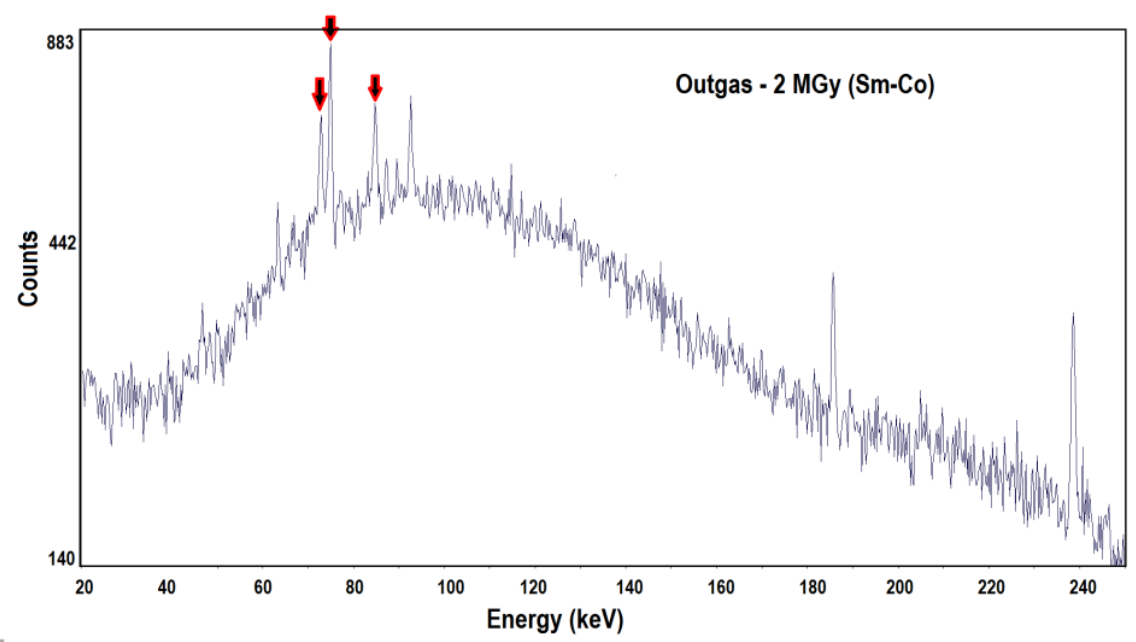

b)

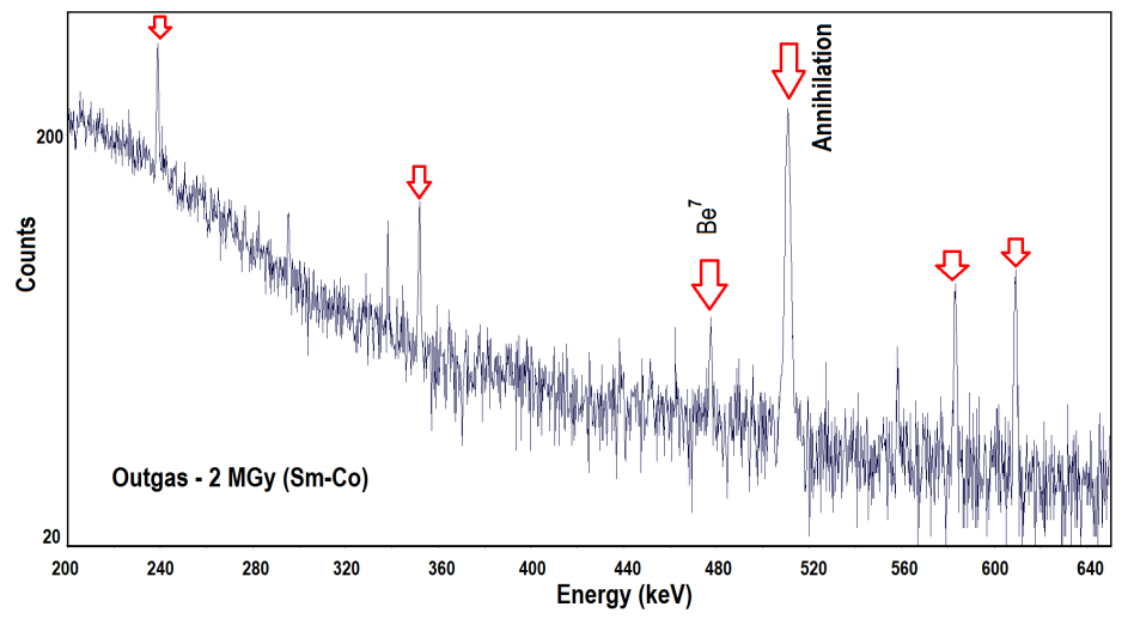

(c)

Figure 15: Photon spectra obtained from: (a) irradiated FF and (b-c) from the outgassing products collected during rotational testing of the FF seal irradiated to $2 \mathrm{MGy}$. Photopeaks of interest are labeled 591 by arrows. 


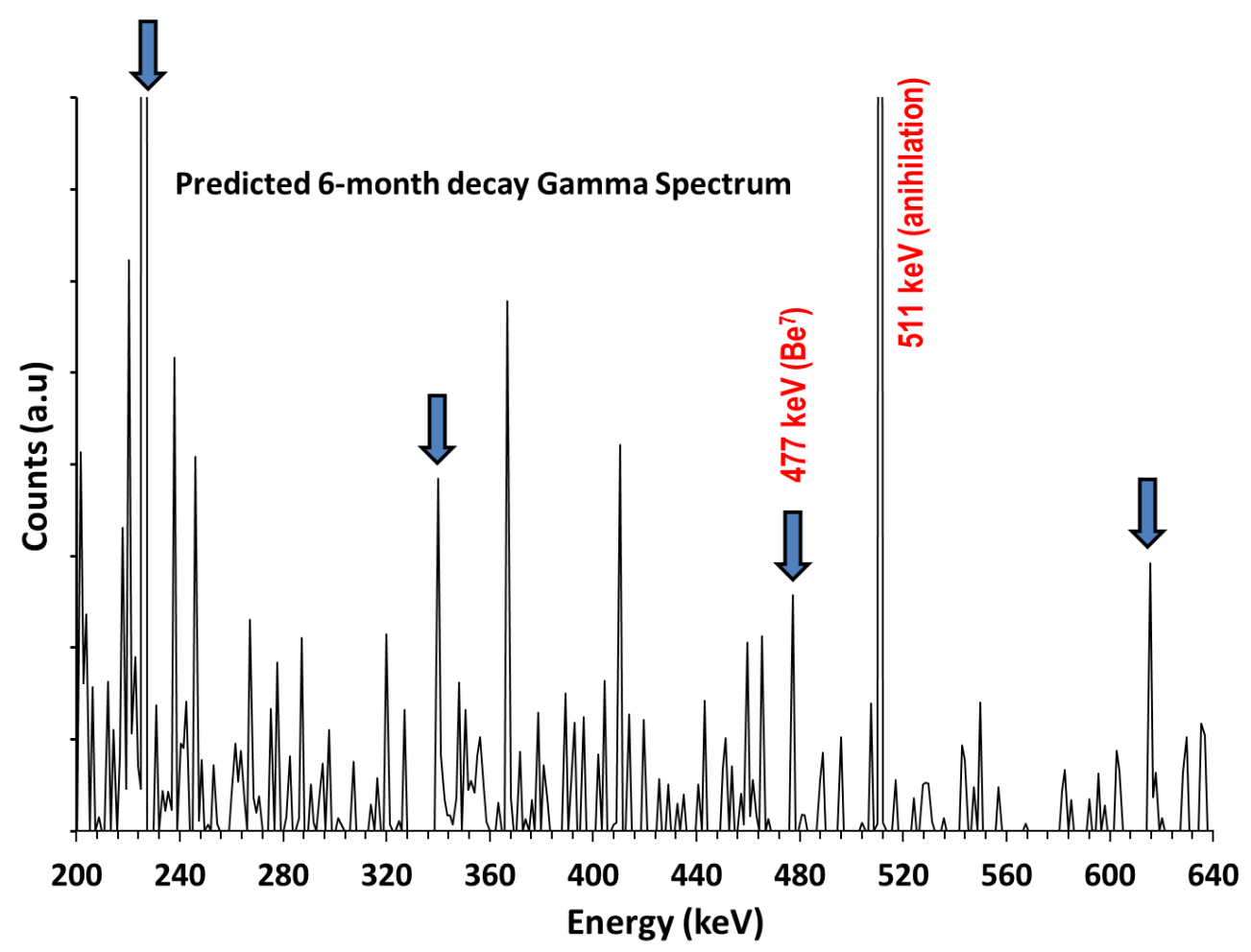

Figure 16: Predicted -spectrum and isotopes in the ferrofluid using the FLUKA model which compares well with actual $\gamma$-spectra shown in Figure 15. Photopeaks of interest are labeled by arrows.

\section{Summary and Conclusion}

The operational performance of ferrofluidic feedthroughs following irradiation up to 20 MGy dose induced by a mixed radiation field resulting from spallation reactions induced by $118 \mathrm{MeV}$ protons at the Brookhaven Linear Isotope Producer beamline was assessed. The irradiation conditions and the mix of irradiating species mimic those expected at the rare isotope production target and the beam dump systems at the Facility for Rare Isotope Beams FRIB. Three FFs were irradiated to 0.2 MGy, 2 MGy and 20 MGy dose levels. The 0.2 MGy FF contained an Nd-Fe-B type rare earth magnet and the 2 and 20 MGy dose FFs contained Sm-Co type magnets. The primary objective was to assess the static and rotational performance up to $5,000 \mathrm{rpm}$ and the potential ferrofluid leakage following anticipated ferrofluid degradation and rare earth magnet de-magnetization from radiation damage.

The comprehensive, multi-faceted study revealed the following:

- The FF irradiated at $0.2 \mathrm{MGy}$ (NdFeB-based) did not experience any leak rate change from static to dynamic conditions up to 5,000 rpm indicating that at this irradiation damage level the ferrofluidic properties (magneto-viscous) remain intact.

- The FF irradiated with 2 MGy (SmCo-based) showed a relative leak rate increase of $\sim 37 \%$. For a ferrofluid radiation-induced viscosity change of that magnitude no significant influence on the FF seal performance under dynamic conditions up to a dose of $2 \mathrm{MGy}$ is observed. Despite of a small leakage detected for the FF irradiated to 2 MGy dose under dynamic conditions (3,600 to 5,100 rpm) the FF seal remained within the operating envelope of the FRIB production target and beam dump systems.

- The FF breakaway torque obtained for the FF irradiated with 2 MGy after a single rotational speed cycle up 5,000 rpm increased about $70 \%$ in comparison to a non-irradiated FF of the same type under the same measurement conditions. 
- Based on the plan to operate the FRIB beam dump and target systems at 600 and 5,000 rpm respectively, the FF breakaway, static and rotational torques of the motor - coupling - FF - target assembly will have no influence on operation. However, the motor drive force must overcome the breakaway rotational torque when starting to rotate the FRIB target and beam dump loads, which can be up to 1,000 times than the operational torque.

- The rotational torque did not increase significantly for the FFs irradiated with 0.2 and 2 MGy respectively in comparison to a non-irradiated FF of the same model.

- Radiation-induced damage in the ferrofluidic feedthrough irradiated to a dose of 20 MGy (SmCobased) rendered the unit inoperable. Breakdown of the ferrofluid occurred at this irradiation level while no de-magnetization of the $\mathrm{Sm}_{2} \mathrm{Co}_{27}$ was observed thus eliminating the presence of a combined damage effect. Therefore an upper dose limit of 2 MGy for the types of tested FFs and under a similar radiation field to the one expected at FRIB was established.

- While previous studies of radiation-induced de-magnetization of $\mathrm{Nd}_{2} \mathrm{Fe}_{14} \mathrm{~B}$ have shown high sensitivity to irradiation accompanied by serious loss of magnetic field intensity, the present study showed that no magnetic field loss occurs up to $0.2 \mathrm{MGy}$. The observation is attributed to the annular shape of the magnet.

- The experiment confirmed the insensitivity to irradiation exhibited by Sm-Co magnets observed in a number of previous studies by showing no loss at 2 and 20 MGy

- Post-irradiation rotational tests revealed ferrofluid magnetization, influencing of the magneto-viscous effect leading to de-sedimentation and de-agglomeration that could have occurred as a results of irradiation. The observations during post-irradiation performance testing agree precisely with previously conducted experiments of ferrofluid magnetization via solid body rotation and most specifically on the observed optimal rotational speed of $\sim 5,000 \mathrm{rpm}$

- Based on findings to-date regarding (a) radiation dose tolerance of FFs (performance degradation in the absorbed dose interval between 2-20 MGy) and (b) the effect of FF magnetic field orientation with respect to the direction of the irradiating field, follow up studies will focus two important parameters, namely, the identification of the threshold dose for both FF types ( $\mathrm{Sm}-\mathrm{Co}$ and $\mathrm{NdFeB}$ ) where performance begins to degrade and the demagnetization sensitivity of both types of FFs with magnetic field orientation during irradiation. Of primary interest will be the confirmation of the insensitivity of Sm-Co magnet type FF to the orientation with the irradiation field. Previous studies have reported $[18,19]$ that $\mathrm{Sm}$-Co permanent magnets show much higher demagnetization resistance than the NdFeB counterparts. Confirmation of their insensitivity to orientation will eliminate design integration constraints.

- The results from a Monte-Carlo based transport code model developed to identify isotopic and $\gamma$ spectra signatures have compared well with actual outgassing $\gamma$-spectra measurements, thus offering confidence in utilizing such codes in in-situ ferrofluidic feedthrough performance assessment and monitoring.

\section{Acknowledgments}

We acknowledge the NSCL technical assistance of Jim Wagner, Jay Pline, Jim Vincent and Andy Thulin in the conception of the post-irradiation torque measurement testing set-up, the technical discussions with Ferrotec Inc. in the person of Tom Black, Li Ping and Perry Barker, and the support of the BLIP facility staff at BNL, in particular Joseph O'Connor (retired). The discussion on the magnet shape effects on demagnetization with Dr. Al Zeller of Michigan State University is greatly appreciated.

This material is based upon work supported by U.S. Department of Energy Office of Science under Cooperative Agreement DE-SC0000661, the State of Michigan and Michigan State University. 


\section{References}

668 [1] D.J., Morrissey, "Status of the FRIB project with a new fragment separator," Journal of Physics: Conference Series 267 (2011) 012001.

[2] F. Pellemoine, W. Mittig, M. Avilov, D. Ippel, J. Lenz, J. Oliva, I. Silverman, D. Youchison and T. Xu, Nucl. Instr. Meth. A, 655 (2011) 3-9.

673 [4] K. Raj, Effect of nuclear radiation on ferrofluids, technical note by Ferrotec Inc. (2009).

674 [5] K. Raj, B. Moskowitz, and S. Tsuda, Indian J. Eng. Mater. Sci. 11 (2004) 241-252

675 [6] Ferrofluidic Vacuum Rotary Feedthroughs, catalogue edited by Ferrotec Inc.

676 [7] A. Yoshida, T. Suda, T. Ohtsuki, H. Yuki and T. Kubo, Nucl. Instr. Meth. A 590 (2008) 204-

[8] J.W. Born, Elastomeric Materials in Radiation Effects on Organic Materials, R.O. Bolt and J.G. Carroll, (1963) Academic Press New York.

[10] R.O. Bolt, Effects of Radiation on Lubricants in CRC Handbook of Lubrification and Tribology, Application and Maintenance, G.E. Totten (2006) CRC Press.

[11] J.J. O'Connor, J. Boyd, and E.A. Avallone, Standard Handbook of Lubrication Engineering, A.S.o.L. Engineers and Editors (1968) McGraw-Hill New York.

[12] P. Kopčanský, J. Černák, T. Tima, A. Zentko, M. Timko and P. Slančo, Journal of. Magnetism and. Magnetic Materials, 85 (1990) 103-106.

[13] M. Devi et al. "Characteristic spectroscopic properties of $\gamma$-irradiated rare-earth oxide-based ferrofluids," Journal of Experimental Nanoscience, 7:5, 586-595, 2012)

[14] J. P. Embs, B. Huke, A. Leschhorn, and M. Lücke, "Equilibrium and Nonequilibrium Behaviour of Ferrofluids - Experiments and Theory," Z. Phys. Chem. 222 (2008) 527-586

[15] Felicia, Leona J.; Vinod, Sithara; Philip, John, "Recent Advances in Magnetorheology of Ferrofluids (Magnetic Nanofluids) - A Critical Review," Journal of Nano-fluids, Vol. 5, Number 1, March 2016, pp. 1-22(22)

[16] A.F. Zeller, "Radiation Damage Mechanisms in NdFeB," Paper No. W 6.2, Eleventh International Workshop on Rare-Earth Magnets and Their Applications, Pittsburg, PA, pp. 2124, 1990.

[17] Kankonen et al., "Effects of proton and a irradiations on permanent magnets," Physical Review B, Vol. 49, No. 9, pp. 6052-6057, 1994

[18] R. D. Brown and J. R. Cost, "Radiation-Induced Changes in Magnetic Properties of Nd-Fe-B Permanent Magnets," IEEE Transactions on Magnetics, Vol. 25. No. 4, pp. $\quad 3117-$ 3120, 1989

[19] Bizen et al., "Radiation Damage in Magnets for Undulators at Low Temperature," Proceedings of EPAC 2004, pp. 2092-2094, 2004

[20] Simos, N., P.K. Job, and N. Mokhov, "An Experimental Study of Radiation-induced Demagnetization of Insertion Device Permanent Magnets," Proceedings of 11th Biennial European Particle Accelerator Conference, EPAC'08. 2008.

[21] J. Liu, P. Vora, P. Dent, M. Walmer, C. Chen, J. Talnagi, S. Wu and M. Harmer, Thermal Stability and Radiation Resistance of Sm-Co based Permanent Magnets, Proceedings of the Space Nuclear Conference, Boston (2007).

[22] N. V. Mokhov, The MARS Code System User's Guide, Fermilab-FN-628 (1995); N. V. Mokhov, S. I. Striganov, MARS15 Overview, in Proc. of Hadronic Shower Simulation Workshop, Fermilab, Sept. 2006 AIP Conf. Proc. 896, pp. 50-60 (2007); N. V. Mokhov et al., Prog. Nucl. Sci. Technol. 4, 496 (2014); http://dx.doi.org/10.15669/pnst.4.496; http://wwwap.fnal.gov/MARS/. 
715

716

717

718

719

720

[23] "The FLUKA Code: Developments and Challenges for High Energy and Medical Applications" T.T. Böhlen, F. Cerutti, M.P.W. Chin, A. Fassò, A. Ferrari, P.G. Ortega, A. Mairani, P.R. Sala, G. Smirnov and V. Vlachoudis, Nuclear Data Sheets 120, 211-214 (2014)

[24] "FLUKA: a multi-particle transport code" A. Ferrari, P.R. Sala, A. Fasso`, and J. Ranft, CERN2005-10 (2005), INFN/TC_05/11, SLAC-R-773

[25] LS-DYNA, Version 9.71, Livermore Software Technology Corporation 